\title{
An annotated check-list of spiders (Arachnida: Aranei) of the Khibiny Mountains, Kola Peninsula, Russia
}

\author{
Аннотированный список видов пауков (Arachnida: Aranei) \\ Хибин, Кольский полуостров, Россия
}

\author{
Anna A. Nekhaeva \\ A.A. Нехаева \\ A.N. Severtsov Institute of Ecology and Evolution, Russian Academy of Sciences, Leninsky pr. 33, Moscow, 119071, Russia. E-mail: \\ Adrealinea@gmail.com \\ Институт проблем экологии и эволюции РАН, Ленинский проспект, 33, Москва 119071 Россия.
}

KEY WORDS: Spiders, Khibiny Mountains, Kola Peninsula, fauna, list of species.

КЛЮЧЕВЫЕ СЛОВА: Пауки, Хибины, Кольский полуостров, фауна, список видов.

ABSTRACT. An annotated check-list of spiders of the Khibiny Mountains (Kola Peninsula, Russia) based on both original and literature-derived data is presented for the first time. The list contains 161 species belonging to 17 families. Twenty species (Araniella proxima, Cyclosa conica, Gnaphosa bicolor, G. lapponum, G. leporina, Zelotes clivicola, Agyneta affinis, A. rurestris, Ceratinella wideri, Dicymbium nigrum, Drapetisca socialis, Entelecara erythropus, Moebelia penicillata, Oedothorax apicatus, Porrhomma campbelli, Agroeca lusatica, Alopecosa pinetorum, A. taeniata, Philodromus cespitum, Titanoeca nivalis) are recorded from Kola Peninsula for the first time. A preliminary analysis of the studied fauna is presented.

РЕЗЮМЕ. Впервые приведен аннотированный список пауков Хибин (Кольский полуостров, Россия), составленный на основе оригинальных сборов и литературных данных. Список включает 161 вид, относящихся к 17 семействам, из которых 20 видов (Araniella proxima, Cyclosa conica, Gnaphosa bicolor, G. lapponum, G. leporina, Zelotes clivicola, Agyneta affinis, A. rurestris, Ceratinella wideri, Dicymbium nigrum, Drapetisca socialis, Entelecara erythropus, Moebelia penicillata, Oedothorax apicatus, Porrhomma campbelli, Agroeca lusatica, Alopecosa pinetorum, A. taeniata, Philodromus cespitum, Titanoeca nivalis) отмечены впервые для Кольского полуострова. Проведен предварительный анализ изученной фауны.

\section{Introduction}

The Khibiny Mts are a small mountain system located in the central part of the Kola Peninsula. The first (and the only) report on spiders of the Khibiny seemed to be V.Y. Fridolin's [1936] monograph devoted to a general account of the Khibiny biota, referring to rep- resentatives of three spider families: Araneidae, Lycosidae and Thomisidae. Unfortunately, the spider material collected by Fridolin was not found neither in the collections of the Zoological Museum of the Moscow State University (Moscow, Russia), nor in the Zoological Institute of the RAS (St. Petersburg, Russia) [K.G. Mikhailov, pers. comm.]. Therefore, it is unclear what spider species were found by Fridolin [1936]. Since that time, there have been no records of spiders from the Khibiny until the first decade of the 21 st century.

At present, all the available data on the spider fauna of the Khibiny Mts and on spider distribution is scattered amongst a number of recently published papers [Tanasevitch, 2008; Vasilyeva, Nekhaev, 2009; Kamayev, 2010; Tanasevitch, Kamayev, 2011; Rybalov, Kamayev, 2012]. The most complete list was published by Tanasevitch \& Kamayev [2011] in the paper devoted to the entire spider fauna of Kola Peninsula in which 77 species from the Khibiny Mts were reported. However, special studies on the Khibiny spider fauna are yet absent.

An extensive newly collected material on spiders from the Khibiny allows us to significantly expand a number of the species known from this region. The aim of the present paper is to give an updated account of the spider fauna of the Khibiny Mts The paper contains new data that were obtained by different authors at different times and by various methods. The analysis of the studied spider fauna, including the UPGMA similarity of different habitats, provided below is to be considered provisional.

Study area, material and methods

The Khibiny massif does not exceed $50 \mathrm{~km}$ in its length. It is limited by Imandra and Umbozero Lakes from the west and east sides respectively (Fig. 1). Flat, dome- and ridge-like tops are typical of the Khibiny 


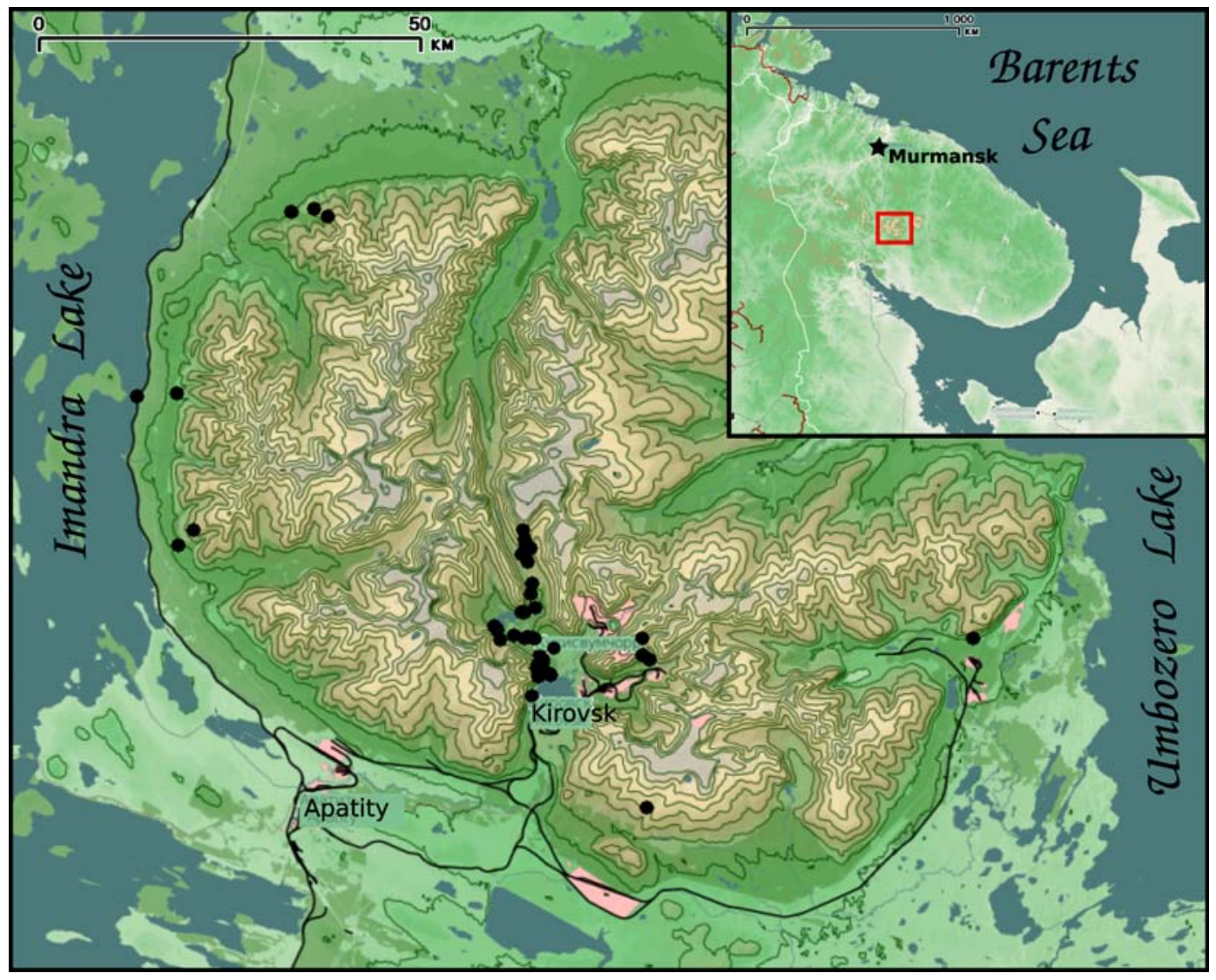

Fig. 1. Map of the Khibiny with the collecting localities.

Рис. 1. Карта Хибин с указанием мест сбора.

(Fig. 2a) [Mishkin, 1953]. The climate of Khibiny Mts differs from the adjacent lowland areas: the winters are warm and settled while the summers are chilly and unsteady [Yakovlev, 1961]. Altitudes vary from 130 to $1200 \mathrm{~m}$ a.s.l. There are three main altitudinal belts in the Khibiny Mts: forest, subalpine and alpine ones [Mishkin, 1953; Shlyakov, 1961]. Position of the borders between the belts varies, depending on a mountain location, slope steepness and its exposition, and many other factors, hence only approximate limits of the belts can be outlined. The forest belt (Fig. 2b) covers the lower parts of slopes up to $260-400 \mathrm{~m}$ a.s.l. and is represented by different types of the spruce-pine forests (the northern taiga communities). The subalpine belt (Fig. 2c) includes the mountain birch forests and occurs at the elevations up to $500 \mathrm{~m}$ a.s.l. The alpine belt (Fig. 2a,d,e) occupies the largest part of the massif and includes the mountain tundra communities up to $850 \mathrm{~m}$ a.s.l. (Fig. 2a,d) and the 'cold stony desert' (Fig. 2e) [Mishkin, 1953] situated on the highest plateau and peaks above $850 \mathrm{~m}$ a.s.l. [Koroleva, 2001; Kremenetski et al., 2004; Zenkova et al., 2011]. The alpine tundra is subdivided into yernik, lichen and moss tundras. Intrazonal communities, such as bogs, screes, cliffs, meadows and so on, exist in all three belts [Mishkin, 1953].

As the apatite-nepheline deposits are located in the Khibiny, the south-eastern part of the massif is strongly damaged by intensive mining operations being performed there since the 1930s (Fig. 2f). Trial and prospecting pits occur in different parts of the mountains.

The spider collecting for the present study was carried out in the Khibiny in September 2008, June, August-September 2009, June-August 2010, JuneAugust 2011, June-July 2013, and June-September 2014. The majority of samples was taken from native habitats. Anthropogenic habitats, including mining excavations, were also studied but less comprehensively as compared to native ones. The most intensive studies were conducted in the south part of the Khibiny Mts (Fig. 1). Spiders were mainly collected by pitfall traps and the sifting of moss and litter. Sweeping, hand collecting and soil sampling were also used (see additional information about the sampling method mentioned below under the description of each sample).

Some preliminary results of this study have already been published [Tanasevitch, 2008; Vasilyeva, Nekhaev, 2009; Kamayev, 2010; Tanasevitch, Kamayev, 2011]. The material is temporary deposited in the personal author collection, and later will partly be deposited to the Zoological Museum of the Moscow State University (ZMMU), Moscow, Russia.

Details of the collecting localities are as given below. For an additional information about the localities on the NE slope of Vudyavrchorr Mt. see Zenkova et 

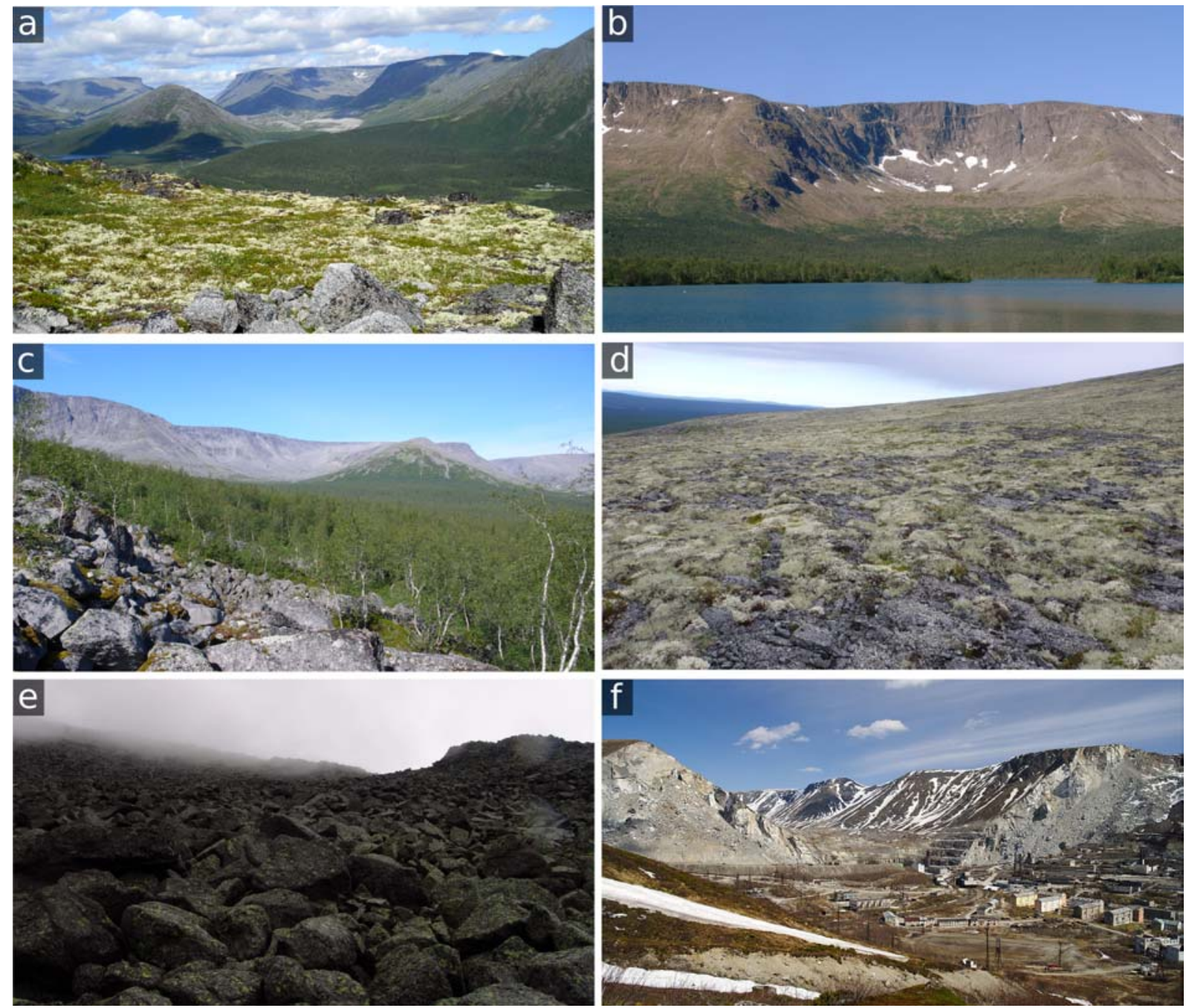

Fig. 2. Khibiny landscapes: a — view of the Malyi Vudyavr Lake valley from Vudyavrchorr Mt. (from left to the right: Takhtarvumchorr Mt., Poachvumchorr Mt., Kukisvumchorr Mt., South Kukisvumchorr Mt.), July 2011; b — the forest belt, Takhtarvumchorr Mt. Range, 2.08.2009 (foto I. Nekhaev) ; c - the subalpine belt, Vudyavrchorr Mt., July 2011 (foto A. Tanasevitch); $\mathrm{d}$ - the alpine belt, Mannepakhk Mt., July 2011 (foto A. Tanasevitch); e — the stony desert, Vudyavrchorr Mt., July 2008 (foto I. Nekhaev); f — the Kirov mining camp, 1.06.2012 (foto I. Nekhaev).

Рис. 2. Ландшафты Хибин: а - вид на долину оз. Малый Вудъявр с г. Вудъяврчорр (слева направо: г. Тахтарвумчорр, г. Поачвумчорр, г. Кукисвумчорр, г. Южный Кукисвумчорр), июль 2011 г.; b - лесной пояс, хребет Тахтарвумчорр, 2.08.2009 (фото И. Нехаева); с - субальпийский пояс, г. Вудъяврчорр, июль 2011 г. (фото А. Танасевича); d - альпийский пояс, г. Маннепахк, июль 2011 г. (фото А. Танасевича); е — каменистая пустыня, г. Вудъяврчорр, июль 2008 г. (фото И. Нехаева); f рудкик им. Кирова, 1.06.2012 (фото И. Нехаева).

al. [2009]. The following abbreviations are used in the text: MSUFS - Field Station of the Lomonosov Moscow State University (Kirovsk-23); PABGI - PolarAlpine Botanical Garden-Institute; A.N. - Anna A. Nekhaeva, A.T. - Andrei V. Tanasevitch, I.K. - Ilya O. Kamayev, I.N. - Ivan O. Nekhaev, I.Z. - Irina V. Zenkova, V.P. - Viktoriya V. Pozharskaya; collecting methods: sift. — the sifting of moss and litter, $\mathrm{p} / \mathrm{t}$ pitfall traps, sw. - sweeping, h/c - hand collecting, s/ s - soil samples.

Localities:

Ai: Aikuaivenchorr Mt., WNW slope (67 $37^{\prime} 29^{\prime \prime}$ N 33 $33^{\circ}$ 43" E), 432-445 $\mathrm{m}$ a.s.1., dwarf shrub mountain birch forest, leg. I.Z.
B-1: Vicinities of Maliy Vudyavr Lake $\left(67^{\circ} 39^{\prime} 46.9^{\prime \prime} \mathrm{N}\right.$ $\left.33^{\circ} 36^{\prime} 31^{\prime \prime} \mathrm{E}\right), 371 \mathrm{~m}$ a.s.1, birch forest with tall grass: Sorbus sp., Juniperus sibirica, Chamerion angustifolium, Solidago sp., Geranium sp., Vaccinium myrtillus, Betula nana, leg. A.T., A.N.

B-2: Vicinities of Maliy Vudyavr Lake $\left(67^{\circ} 39^{\prime} 52.2^{\prime \prime} \mathrm{N}\right.$ $\left.33^{\circ} 36^{\prime} 15.5^{\prime \prime} \mathrm{E}\right), 365 \mathrm{~m}$ a.s.1., birch forest, leg. A.T., A.N.

Bog: Carex-Sphagnum bog at the foot of Vudyavrchorr

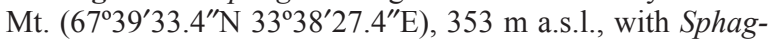
num sp., C. angustifolium, B. nana, Salix sp., Carex rastrata, Eriophorum vaginatum, sometimes with open water areas, leg. A.T., A.N

Bot-1: PABGI vicinity $\left(67^{\circ} 39^{\prime} 18.5^{\prime \prime} \mathrm{N} 33^{\circ} 40^{\prime} 26.2^{\prime \prime} \mathrm{E}\right)$, meadow with Heracleum sosnowskyi, Anthriscus sylvestris, Urtica dioica, Salix sp., leg. A.T., A.N. 
Bot-2: PABGI vicinity $\left(67^{\circ} 39^{\prime} 32.3^{\prime \prime} \mathrm{N} 33^{\circ} 39^{\prime} 6.1^{\prime \prime} \mathrm{E}\right), 393$ $\mathrm{m}$ a.s.1., spruce sod-dwarf shrub forest with Picea obovata, Pinus sylvestris, B. subarctica, Sorbus sp., Chamaepericlymenum suecicum, Solidago lapponica, V. myrtillus, Dicranum sp., Barbilophozia lycopodioides, under bark of birch, leg. A.N.

Bot-3: PABGI vicinity, under bark of spruce, leg. A.N.

Bot-4: PABGI vicinity, a glade in spruce forest with Picea sp., Betula sp., Sphagnum sp., Equisetum sp., leg. A.N.

Bot-5: PABGI vicinity $\left(67^{\circ} 39^{\prime} 35.8^{\prime \prime} \mathrm{N} 33^{\circ} 38^{\prime} 42.5^{\prime \prime} \mathrm{E}\right)$, $364 \mathrm{~m}$ a.s.l., rocky scree near the road, leg. A.T., A.N.

Im-1: Vicinities of the railway station Imandra $\left(67^{\circ}\right.$ $\left.50^{\prime} 54.2^{\prime \prime} \mathrm{N} 33^{\circ} 22^{\prime} 2.6^{\prime \prime} \mathrm{E}\right), 284 \mathrm{~m}$ a.s.l., dwarf shrub-mosslichen pine forest with $P$. sylvestris, $B$. subarctica, $P$. obovata, Salix caprea, Ledum palustre, Linnaea borealis, Empetrum hermaphroditum, Avenella flexuosa, V. myrtillus, $V$. uliginosum, V. vitis-idaea, Hylocomium splendens, Pleurozium schreberi, under bark of pine and from spiderweb, leg. A.T., A.N.

Im-2: Vicinities of the railway station Imandra, Mt. Mannepakhk, NW slope (67 $\left.50^{\prime} 46^{\prime \prime} \mathrm{N} 33^{\circ} 24^{\prime} 32.7^{\prime \prime} \mathrm{E}\right), 555 \mathrm{~m}$ a.s.l., patchy lichen tundra with boulders outcrops (moss absent), leg. A.T., A.N.

Im-3: Vicinities of the railway station Imandra, Mt Mannepakhk, NW slope (67 $\left.50^{\prime} 57.6^{\prime \prime} \mathrm{N} 33^{\circ} 23^{\prime} 35.3^{\prime \prime} \mathrm{E}\right), 431$ m a.s.l., dwarf shrub-lichen tundra with Arctostaphylos uvaursi, B. nana, E. hermaphroditum, Arctous alpina, Juncus trifidus, Alectoria ochroleuca, Cladina arbuscula, Cl. stellaris, Flavocetraria cucullata, F. nivallis, leg. A.T., A.N.

K-1: Kukisvumchorr Mt. (67 $\left.42^{\prime} 2.4^{\prime \prime} \mathrm{N} 33^{\circ} 38^{\prime} 31.3^{\prime \prime} \mathrm{E}\right)$, $693 \mathrm{~m}$ a.s.1., lichen tundra with B. nana, Cetraria spp., Cladonia spp., Empetrum sp., leg. I.N.

K-2: Kukisvumchorr Mt. $\left(67^{\circ} 42^{\prime} 12.6^{\prime \prime} \mathrm{N} 33^{\circ} 38^{\prime} 23.8^{\prime \prime} \mathrm{E}\right)$, $792 \mathrm{~m}$ a.s.l., among stones, leg. I.N.

K-3: Kukisvumchorr Mt. $\left(67^{\circ} 42^{\prime} 26.7^{\prime \prime} \mathrm{N} 33^{\circ} 38^{\prime} 17.3^{\prime \prime} \mathrm{E}\right)$, $882 \mathrm{~m}$ a.s.l., on stones, leg. I.N.

K-4: A hollow between the S slope of Kukisvumchorr Mt. and moraine $\left(67^{\circ} 41^{\prime} 47^{\prime \prime} \mathrm{N} 33^{\circ} 38^{\prime} 14.3^{\prime \prime} \mathrm{E}\right), 288 \mathrm{~m}$ a.s.1., sparse dwarf shrubs, damp, leg. I.N.

K-be: Near the open pit at the foot of Southern Kukisvumchorr Mt. $\left(67^{\circ} 41^{\prime} 1.4^{\prime \prime} \mathrm{N} 33^{\circ} 38^{\prime} 57.9^{\prime \prime} \mathrm{E}\right), 383 \mathrm{~m}$ a.s.1., mountain birch forest with Betula spp., Salix spp., Juniperus sp., Vaccinium spp., Empetrum sp., Chamaenerion angustifolium, Cetraria spp., Cladonia spp., sand, broken stone, leg. I.N.

K-It: Moraine ridge near the S slope of Kukisvumchorr Mt. $\left(67^{\circ} 41^{\prime} 35.5^{\prime \prime} \mathrm{N} 33^{\circ} 38^{\prime} 37.8^{\prime \prime} \mathrm{E}\right), 497 \mathrm{~m}$ a.s.l., lichen tundra with Cetraria spp., Cladonia spp., B. nana, leg. I.N.

K-lut1: Kukisvumchorr Mt. $\left(67^{\circ} 41^{\prime} 53.1^{\prime \prime} \mathrm{N} 33^{\circ} 38^{\prime}\right.$ 29.9"E), $592 \mathrm{~m}$ a.s.1., lichen-dwarf shrub mountain tundra with Cetraria spp., Cladonia spp., V. vitis-idaea, leg. I.N.

K-lut2: Kukisvumchorr Mt., S slope $\left(67^{\circ} 41^{\prime} 56.6^{\prime \prime} \mathrm{N}\right.$ $\left.33^{\circ} 38^{\prime} 55.1^{\prime \prime} \mathrm{E}\right), 560 \mathrm{~m}$ a.s.1., lichen-dwarf shrub mountain tundra with Cladonia spp., Cetraria spp., Vaccinium spp., Empetrum sp., leg. A.N., I.N.

K-lut3: Kukisvumchorr Mt., S slope $\left(67^{\circ} 41^{\prime} 53.3^{\prime \prime} \mathrm{N}\right.$ $\left.33^{\circ} 38^{\prime} 31.7^{\prime \prime} \mathrm{E}\right), 590 \mathrm{~m}$ a.s.1., lichen-dwarf shrub mountain tundra with Cladonia spp., Cetraria spp., Vaccinium spp., Empetrum sp. and stony areas without vegetation, leg. A.N., I.N.

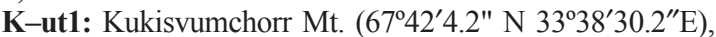
$711 \mathrm{~m}$ a.s.l., dwarf shrub tundra, among the stones, leg. I.N.

K-ut2: Kukisvumchorr Mt., S slope $\left(67^{\circ} 42^{\prime} 4^{\prime \prime} \mathrm{N}\right.$ $\left.33^{\circ} 38^{\prime} 34^{\prime \prime} \mathrm{E}\right), 705 \mathrm{~m}$ a.s.1., dwarf shrub mountain tundra with Vaccinium spp., Empetrum sp., Juniperus sp., B. nana, Cladonia spp., Cetraria spp., leg. A.N., I.N.
Lo-ad: Lovchorr Mt., S slope, Vicinities of meteorological station, 1065-1093 m a.s.l., high-mountain stone desert with sparse clumps of grass, mosses and lichens with Saxifraga oppositifolia, Luzula arcuata, Harrimanella hypnoides, $S$. reticulata, Oxyria digyna, Dryas punctata, C. bigelowii, Cetraria spp., Cladonia spp., leg. I.Z., V.P.

Lo-be: Lovchorr Mt., N slope, $452 \mathrm{~m}$ a.s.1., mountain birch forest with $B$. czerepanovii, B. subarctica, Picea sp., Vaccinium spp., E. hermaphroditum, Phyllodoce caerulea, S. glauca, Arctostaphylos sp., S. lapponica, A. flexuosa, Lycopodium annotinum, Diphasiastrum alpinum, Nephroma spp., Cetraria spp., Cladonia spp., leg. I.Z., V.P.

Lo-lumt: Lovchorr Mt., S slope $\left(67^{\circ} 35^{\prime} \mathrm{N} 33^{\circ} 47^{\prime} \mathrm{E}\right), 735$ $\mathrm{m}$ a.s.1., lichen-dwarf shrub-moss mountain tundra with $\mathrm{Cla}$ donia rangiferina, $F$. nivalis, Cetraria islandica, E. hermaphroditum, Vaccinium spp., A. alpina, Poa alpina, D. punctata, Andromeda polifolia, B. nana, Racomitrium spp., P. schreberi, Festuca ovina, C. bigelowii, Antennaria dioica, leg. I.Z., V.P.

MSU: The road near MSUFS, sand, stones, leg. I.N.

Nef-1: Vicinities of the railway station Nefelinovye Peski $\left(67^{\circ} 46^{\prime} 3.1^{\prime \prime} \mathrm{N} 33^{\circ} 14^{\prime} 0.2^{\prime \prime} \mathrm{E}\right), 287 \mathrm{~m}$ a.s.1., SW slope of Khibinpakhkchorr Mt., bilberry-moss-lichen pine forest with $P$. sylvestris, $B$. subarctica, $L$. borealis, $E$. hermaphroditum, $V$. myrtillus, V. vitis-idaea, Dicranum scoparium, Racomitrium microcarpon, $H$. splendens, C. stellaris, C. arbuscula, C. rangiferina, C. crispata, leg. A.T., A.N.

Nef-3: Vicinities of the railway station Nefelinovye Peski, stony bank of Imandra Lake, opposite to Vysokiy Island $\left(67^{\circ} 45^{\prime} 58.8^{\prime \prime} \mathrm{N} 33^{\circ} 11^{\prime} 11.3^{\prime \prime} \mathrm{E}\right), 127 \mathrm{~m}$ a.s.1., under stones and trash, leg. A.T., A.N.

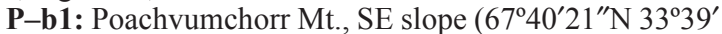
$\left.11^{\prime \prime E}\right), 398 \mathrm{~m}$ a.s.l., moss-grass birch forest (mountain birch forest) with Sorbus sp., Picea sp., Salix sp., J. sibirica, V. myrtillus, Ch. suecicum, Deschampsia cespitosa, Gymnocarpium dryopteri, P. alpina, C. angustifolium, Anthoxanthum alpinum, Politrichum spp., S. lapponica, H. hypnoides, Melampyrum sylvaticum, Hieracium alpinum, L. annotinum, Trientalis europaea, leg. I.Z., V.P.

P-b2: Poachvumchorr Mt., S slope $\left(67^{\circ} 40^{\prime} 15^{\prime \prime} \mathrm{N} 33^{\circ}\right.$ $38^{\prime} 16^{\prime \prime} \mathrm{E}$ ), $390-420 \mathrm{~m}$ a.s.1., grass birch forest (mountain birch forest) with Betula sp., Sorbus sp., Salix sp., V. vitisidaea, V. uliginosum, V. myrtillus, E. hermaphroditum, Calluna vulgaris, Cirsium heterophyllum, $F$. ovina, $P$. nemoralis, Ranunculus sp., M. pratense, Geranium sylvaticum, Solidago sp., Dicranum sp., Racomitrium sp., Polytrichum sp., Cetraria spp., Cladina spp., Cladonia spp., leg. I.Z., V.P.

P-p: Poachvumchorr Mt., S slope $\left(67^{\circ} 40^{\prime} 14^{\prime \prime} \mathrm{N} 33^{\circ} 38^{\prime}\right.$ 29"E), $395 \mathrm{~m}$ a.s.1., grass-moss-dwarf shrub spruce forest (open spruce forest) with Picea sp., Betula sp., Sorbus sp., Rubus saxatilis, Euphrasia sp., V. vitis-idaea, V. myrtillus, E. hermaphroditum, M. pratense, M. sylvaticum, Ch. suecicum, G. dryopteris, A. dioica, F. ovina, P. nemoralis, Pyrola rotundifolia, Milium effusum, Agrostis sp., S. lapponica, Sphagnum sp., Mnium sp., Dicranum sp., Racomitrium sp., Polytrichum sp., Nephroma sp., Cetraria sp., Cladina sp., Cladonia sp., leg. I.Z., V.P.

P-t: Northern micro-slope of Poachvumchorr Mt., SE slope $\left(67^{\circ} 40^{\prime} 44^{\prime \prime} \mathrm{N} 33^{\circ} 38^{\prime} 53^{\prime \prime} \mathrm{E}\right), 411 \mathrm{~m}$ a.s.l., mountain dwarf shrub tundra with $F$. nivalis, $C$. islandica, $C l$. rangiferina, Cladonia sp., A. nigricans, B. nana, C. vulgaris, V. vitisidaea, $V$. uliginosum, $V$. myrtillus, E. hermaphroditum, $A$. alpina, Nardus stricta, Polytrichum spp., C. bigelowii, $H$. hypnoides, J. trifidus, Loiseleuria sp., leg. I.Z., V.P.

SU-1: Suolayv Mt., NW slope $\left(67^{\circ} 39^{\prime} 32^{\prime \prime} \mathrm{N} 34^{\circ} 10^{\prime} 03^{\prime \prime} \mathrm{E}\right)$, 380-387 m a.s.l., moss-dwarf shrub mountain tundra, leg. I.Z. 


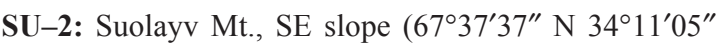
E), 270-308 $\mathrm{m}$ a.s.1., dwarf shrub-rich in herbs mountain birch forest, leg. I.Z.

SU-3: Suolayv Mt., SE slope $\left(67^{\circ} 39^{\prime} 37^{\prime \prime}\right.$ N 34 $14^{\circ} 06^{\prime \prime}$ E), 250-270 m a.s.l., grassy mountain spruce forest, leg. I.Z.

V: Vudyavrchorr Mt., rocks, dwarf shrubs (Empetrum sp.), leg. I.N.

V-be1: Vudyavrchorr Mt., NE slope $\left(67^{\circ} 38^{\prime} 42.6^{\prime \prime} \mathrm{N}\right.$ $\left.33^{\circ} 39^{\prime} 26.9^{\prime \prime} \mathrm{E}\right), 372 \mathrm{~m}$ a.s.1., mountain birch forest with Betula sp., B. nana, E. hermaphroditum, Salix spp., V. myrtilis, H. splendens, P. schreberi, leg. I.K.

V-be2: Vudyavrchorr Mt., NE slope $\left(67^{\circ} 38^{\prime} 49^{\prime \prime} \mathrm{N} 33^{\circ}\right.$ $\left.39^{\prime} 24^{\prime \prime} \mathrm{E}\right), 419 \mathrm{~m}$ a.s.1., mountain birch forest with $B . c z$ erepanovii, B. subarctica, Sorbus gorodkovii, V. mirtillus, E. hermaphroditum, A. polifolia, G. sylvaticum, Geum rivale, P. rotundifolia, Dicranum spp., Marchantia polimorpha, leg. I.Z., V.P.

V-bs: Vudyavrchorr Mt., NE slope $\left(67^{\circ} 38^{\prime} 59^{\prime \prime} \mathrm{N} 33^{\circ} 39^{\prime}\right.$ 43"E), $346 \mathrm{~m}$ a.s.1., Geranium-ferns birch forest with $B$. czerepanovii, S. gorodkovii, G. sylvaticum, G. dryopteris, Ch. suecicum, Barbilophozia spp., leg. I.K.

V-lut1: Vudyavrchorr Mt., NE slope $\left(67^{\circ} 38^{\prime} 42^{\prime \prime} \mathrm{N} 33^{\circ}\right.$ $\left.39^{\prime} 26^{\prime \prime} \mathrm{E}\right), 478 \mathrm{~m}$ a.s.1., lichen-dwarf shrub mountain tundra with $C$. rangiferina, $C$. islandica, $S$. reticulata, $S$. polaris, $E$. hermaphroditum, Vaccinium spp., D. punctata, H. hypnoides, Silene acaulis, $H$. alpinum, $F$. ovina, leg. I.Z., V.P.

V-lut2: Vudyavrchorr Mt., NE slope $\left(67^{\circ} 38^{\prime} 33.5^{\prime \prime} \mathrm{N} 33^{\circ}\right.$ 39'31.6"E), $548 \mathrm{~m}$ a.s.l., mountain dwarf shrub-lichen tundra with E. hermaphroditum, Cladonia spp., F. nivalis, Alectoria spp., Cetraria spp., leg. I.K.

V-pe1: Vudyavrchorr Mt., NE slope $\left(67^{\circ} 39^{\prime} 06^{\prime \prime} \mathrm{N} 33^{\circ} 39^{\prime}\right.$ $\left.33^{\prime \prime} \mathrm{E}\right), 330 \mathrm{~m}$ a.s.1., tall herbs and grass-dwarf shrub-moss spruce forest with $P$. obovata, B. czerepanovii, V. myrtillus, A. flexuosa, Deschampsia cespitosa, P. schreberi, H. splendens, leg. I.K.

V-pe2: Vudyavrchorr Mt., NE slope $\left(67^{\circ} 38^{\prime} 53^{\prime \prime} \mathrm{N} 33^{\circ}\right.$ $\left.39^{\prime} 45^{\prime \prime} \mathrm{E}\right), 357 \mathrm{~m}$ a.s.l., spruce forest with $P$. obovata, $B$. czerepanovii, S. gorodkovii, Vaccinium spp., E. hermaphroditum, G. dryopteris, Cicerbita alpina, C. angustifolium, Melampyrum spp., Polytrichum spp., Sphagnum spp., Dicranum spp., leg. I.Z., V.P.

V-ut1: Vudyavrchorr Mt., NE slope $\left(67^{\circ} 38^{\prime} 43^{\prime \prime} \mathrm{N} 33^{\circ} 39^{\prime}\right.$ $\left.20^{\prime \prime} \mathrm{E}\right), 430 \mathrm{~m}$ a.s.l., dwarf shrub mountain tundra with $A$. alpina, E. hermaphroditum, Vaccinium spp., Loiseleuria procumbens, Diapensia lapponica, C. islandica, Cladonia spp., Alectoria spp., Stereocaulon spp., B. nana, Salix spp., leg. I.Z., V.P.

V-ut2: Vudyavrchorr Mt., NE slope $\left(67^{\circ} 38^{\prime} 42.6^{\prime \prime} \mathrm{N} 33^{\circ}\right.$ $39^{\prime} 26.9^{\prime \prime} \mathrm{E}$ ), $498 \mathrm{~m}$ a.s.1., B. nana-dominated mountain tundra or dwarf shrub-moss tundra with B. nana, E. hermaphroditum, Salix spp., V. myrtillus, H. splendens, P. schreberi, leg. I.K.

VL: Near Vudyavr Lake, forest, dwarf shrubs, under trees, leg. I.N.

VN-1: An open pit at the foot of Vudyavrchorr Mt. $\left(67^{\circ} 39^{\prime} 36.8^{\prime \prime} \mathrm{N} 33^{\circ} 37^{\prime} 43.6^{\prime \prime} \mathrm{E}\right), 345 \mathrm{~m}$ a.s.l., among the stones and sand, sparse Papaver radicatum, C. angustifolium, Juncus sp., S. virgaurea, Salix spp., Betula sp., Juniperus communis, S. caprea, J. trifidus, Astragalus sp., leg. A.T., A.N.

VN-2: The lower part of $\mathrm{N}$ slope of Vudyavrchorr Mt. $\left(67^{\circ} 39^{\prime} 43.8^{\prime \prime} \mathrm{N} 33^{\circ} 36^{\prime} 31.7^{\prime \prime} \mathrm{E}\right), 371 \mathrm{~m}$ a.s.l., dwarf shrubgrass birch forest with Betula sp., B. czerepanovii, J. sibirica, A. flexuosa, E. hermaphroditum, S. lapponica, V. myrtillus, B. lycopodioides, Ptilidium ciliare, Cladonia spp. and mossy boulders, leg. A.T., A.N.

VN-3: Vudyavrchorr Mt., N slope $\left(67^{\circ} 39^{\prime} 28.9^{\prime \prime} \mathrm{N} 33^{\circ}\right.$ $\left.36^{\prime} 41.3^{\prime \prime} \mathrm{E}\right), 496 \mathrm{~m}$ a.s.1., dwarf shrub-lichen mountain tundra with $B$. czerepanovii, B. nana, E. hermaphroditum, A. alpina, $V$. uliginosum, Andreaea rupestris, A. ochroleuca, F. nivalis, F. cuculata, Umbilicaria sp., Cetraria sp., leg. A.T., A.N.

VS-be: Vudyayvrchorr Mt., NE slope $\left(67^{\circ} 38^{\prime} 36.4^{\prime \prime} \mathrm{N}\right.$ $\left.33^{\circ} 40^{\prime} 10.9^{\prime \prime} \mathrm{E}\right), 320 \mathrm{~m}$ a.s.1., tall herbs birch forest with $B$. czerepanovii, Ch. suecicum, C. alpina, Calamagrostis sp., Barbilophozia sp., leg. I.K.

VS-ut: Vudyayvrchorr Mt., S slope $\left(67^{\circ} 38^{\prime} \mathrm{N} 33^{\circ} 39^{\prime} \mathrm{E}\right)$, $333 \mathrm{~m}$ a.s.l., dwarf shrub mountain tundra with $V$. vitis-idaea, E. hermaphroditum, P. caerulea, A. uva-ursi, C. rangiferina, F. nivalis, Nephroma arcticum, J. sibirica, leg. I.Z., V.P.

Vu-1: Vicinities of Kirovsk town, NW coast of Bolshoy Vudyavr Lake $\left(67^{\circ} 38^{\prime} 33.6^{\prime \prime} \mathrm{N} 33^{\circ} 40^{\prime} 13.3^{\prime \prime} \mathrm{E}\right), 334 \mathrm{~m}$ a.s.l., among a stones and patches of vegetation, with J. filiformis, Poa sp., Rorippa palustris, leg. A.T., A.N.

Vu-2: Vicinities of Kirovsk, NW coast of Bolshoy Vudyavr Lake $\left(67^{\circ} 38^{\prime} 33.6^{\prime \prime} \mathrm{N} 33^{\circ} 40^{\prime} 13.3^{\prime \prime} \mathrm{E}\right), 334 \mathrm{~m}$ a.s.l., coastal meadow with Ranunculus repens, D. cespitosa, C. rostrata, Er. vaginatum, J. filiformis, R. palustris, Rumex sp., Agrostis sp., Stellaria palustris, Marchantia sp., leg. A.T., A.N.

Vu-3: Vicinities of Kirovsk, NW coast of Bolshoy Vudyavr Lake $\left(67^{\circ} 38^{\prime} 33.6^{\prime \prime} \mathrm{N} 33^{\circ} 40^{\prime} 13.3^{\prime \prime} \mathrm{E}\right), 334 \mathrm{~m}$ a.s.l., willow-bed on coast with $C$. heterophyllum, $S$. virgaurea, $C$. angustifolium, B. nana, Tussilago farfara, Calamagrostis sp., D. cespitosa, S. palustris, R. repens, R. palustris, $J$. filiformis, $V$. uliginosum, leg. A.T., A.N.

$\mathbf{V u}-\mathbf{4}$ : Vudyavryok river bank, near the bridge in PABGI, leg. A.N.

VNE-1: Vudyavrchorr Mt., NE slope $\left(67^{\circ} 38^{\prime} 34.1^{\prime \prime} \mathrm{N}\right.$ $\left.33^{\circ} 39^{\prime} 30.8^{\prime \prime} \mathrm{E}\right), 538 \mathrm{~m}$ a.s.1., lichen-dwarf shrub mountain tundra, leg. A.T., A.N.

VNE-2: Vudyavrchorr Mt., NE slope $\left(67^{\circ} 38^{\prime} 33.4^{\prime \prime} \mathrm{N}\right.$ $\left.33^{\circ} 39^{\prime} 31.3^{\prime \prime} \mathrm{E}\right), 548 \mathrm{~m}$ a.s.l., spot of moss-dwarf shrub in the middle of lichen tundra with B. nana, Empetrum sp., $V$. myrtillus, $V$. vitis-idaea, A. alpina, $P$. schreberi, Dicranum sp., H. splendens, leg. A.T., A.N.

VNE-3: Vudyavrchorr Mt., NE slope $\left(67^{\circ} 38^{\prime} 31.3^{\prime \prime} \mathrm{N}\right.$ $\left.33^{\circ} 39^{\prime} 27.2^{\prime \prime} \mathrm{E}\right), 571 \mathrm{~m}$ a.s.l., lichen-dwarf shrub mountain tundra with $V$. uliginosum, E. hermaphroditum, B. nana, Cladonia sp., Cetraria sp., Juncus sp., leg. A.T., A.N.

VNE-4: Vudyavrchorr Mt., NE slope $\left(67^{\circ} 38^{\prime} 30.8^{\prime \prime} \mathrm{N}\right.$ $\left.33^{\circ} 39^{\prime} 25.9^{\prime \prime}\right)$, $579 \mathrm{~m}$ a.s.l., patch of Betula nana-lichenmoss tundra with P. schreberi, Cetraria sp., Cladonia sp., Dicranum sp., V. uliginosum, V. vitis-idaea, E. hermaphroditum, B. nana, Salix sp., leg. A.T., A.N.

VNE-5: Vudyavrchorr Mt., NE slope $\left(67^{\circ} 38^{\prime} 51.2^{\prime \prime} \mathrm{N}\right.$ $\left.33^{\circ} 39^{\prime} 27.6^{\prime \prime} \mathrm{E}\right), 400 \mathrm{~m}$ a.s.l., spruce-birch forest with Picea sp., Betula sp., V. myrtillus, Solidago sp., moss, leg. A.T., A.N.

YU-1: Yumechorr Mt., SW slope $\left(67^{\circ} 42^{\prime} 03^{\prime \prime} \mathrm{N} 33^{\circ} 14^{\prime}\right.$ $\left.06^{\prime \prime} \mathrm{E}\right), 314 \mathrm{~m}$ a.s.l., bilberry pine forest, with $P$. sylvestris, V. myrtillus, leg. I.Z.

YU-2: Yumechorr Mt., SW slope, $430 \mathrm{~m}$ a.s.1., dwarf shrub mountain birch forest with $B$. pubescens, leg. I.Z.

YU-3: Yumechorr Mt., SW slope $\left(67^{\circ} 42^{\prime} 27^{\prime \prime} \mathrm{N} 33^{\circ} 15^{\prime}\right.$ $\left.10^{\prime \prime} \mathrm{E}\right), 500 \mathrm{~m}$ a.s.1., dwarf shrub-lichen mountain tundra, leg. I.Z.

YUK-1: Yuksporr Mt., SE slope $\left(67^{\circ} 38^{\prime} 59^{\prime \prime} \mathrm{N} 33^{\circ}\right.$ $\left.47^{\prime} 17^{\prime \prime} \mathrm{E}\right), 460-490 \mathrm{~m}$ a.s.1., dwarf shrub mountain birch forest, leg. I.Z.

YUK-2: Yuksporr Mt., SE slope $\left(67^{\circ} 39^{\prime} 06^{\prime \prime} \mathrm{N} 33^{\circ} 46^{\prime}\right.$ 44"E), 625-645 m a.s.l., dwarf shrub-lichen mountain tundra on the slope, leg. I.Z.

YUK-3: Yuksporr Mt., SE slope, $\left(67^{\circ} 39^{\prime} 32^{\prime \prime} \mathrm{N} 33^{\circ} 46^{\prime}\right.$ $\left.44^{\prime \prime} \mathrm{E}\right), 710-730 \mathrm{~m}$ a.s.l., dwarf shrub-lichen mountain tundra on the plateau, leg. I.Z. 
The bulk of the material collected was identified by the author. The species identifications of the material collected by Ilya Kamayev were kindly provided to us by the latter colleague.

The recorded taxa are listed in the alphabetic order. The nomenclature generally follows that of World Spider Catalog [2015]; any differences/changes as compared to the WSC are marked in the text. The spider species that have been recorded to the Kola Peninsula for the first time are marked with $(*)$; the species that have not been found during our investigation are marked with $\left({ }^{\wedge}\right)$. The codes given in square brackets correspond to the aforementioned localities. The findings of juveniles have been included in the list only if the adults of these taxa were not collected.

Unfortunately, spiders from the localities V-lut1, V-ut1, V-be2, and V-pe2, which were collected in September 2008 and June 2009 by different sampling methods, were mixed up during previous sorting. For these lots the dates and collecting methods are not listed.

Distributional ranges of the species are given according to Ivie [1969], Saaristo [1971], Leech [1972], Palmgren [1975; 1976; 1977], Dondale, Redner [1975; 1978; 1990], Ovtsharenko et al. [1992], Platnick, Dondale [1992], Eskov [1994], Esyunin, Efimik [1996], Saaristo, Eskov [1996], Logunov, Marusik [2000], Marusik et al. [2000; 2006; 2007], Azarkina, Logunov [2001], Marusik, Koponen [2005], Esyunin [2006], Tuneva [2006], Tanasevitch, Koponen [2007], Tanasevitch [2008; 2010; 2011; 2013; 2014], Marusik, Eskov [2009], Marusik, Alfimov [2012], Blagoev et al. [2013], Esyunin et al. [2013], Mikhailov [2013], Kronestedt et al. [2014], Marusik, Omelko [2014].

In the Discussion section and Table 1, all the studied communities are indicated as follows: the communities predominated by Picea sp. are named "spruce forests", those predominated by Betula spp. — "birch forests" (except for the "mountain birch forests"), all the mountain tundra communities are named «tundra». Furthermore, the spiders that were collected from the Yuksporr Mts (YUK-1; YUK-2; YUK-3), Yumechorr (YU-1; YU-2; YU-3), Suolayv (SU-1; SU-2; SU-3), and Aikuaivenchorr (Ai) in 2013 and 2014 are not listed in Table 1 because the material has not been fully processed.

Faunistic similarities between the spider faunas of various biotopes have been calculated by the Dice coefficient in PAST 1.97 [Hammer et al., 2001]. A dendrogram has been plotted by the UPGMA algorithm.

\section{List of species}

\section{Fam. AMAUROBIIDAE (1)}

Arctobius agelenoides (Emerton, 1919)

PREVIOUS RECORDS. Vudyavrchorr Mt. [Tanasevitch, Kamayev, 2011]

MATERIAL. $1 \sigma^{\top}, 1$, [V-ut1]; 1 , [V-lut2], sift., h/c, VIII.2010; $1 \sigma^{7}$, [VNE-1], sift., 25.VII.2011.

RANGE. Palaearctic-W Nearctic arcto-boreal.
Fam. ARANEIDAE (2)

Araniella proxima (Kulczyński, 1885) *

MATERIAL. $1 \sigma^{7}$, [SU-3], p/t, 22.06-10.07.2013.

RANGE. Holarctic boreal.

Cyclosa conica (Pallas, 1772) *

MATERIAL. 3 juv, [Im-1], h/c, 30.VII.2011.

RANGE. Sibcircum-Holarctic boreo-nemoral.

\section{Fam. CLUBIONIDAE (1)}

Clubiona kulczynskii Lessert, 1905

MATERIAL. $1 \mathrm{O}^{2}$, [Ai], p/t, 21.06-8.07.2013.

RANGE. Holarctic boreo-nemoral.

Fam. DICTYNIDAE (1)

Dictyna uncinata Thorell, 1856

MATERIAL. $1 \sigma^{\top}$ [SU-2], p/t, 22.06.-10.07.2013, $1 \sigma^{7}$ [SU3], p/t, 22.06.-10.07.2013.

RANGE. Palaearctic boreo-nemoral.

\section{Fam. GNAPHOSIDAE (10)}

Gnaphosa bicolor (Hahn, 1833) *

MATERIAL. 1 \%, [YU-1], p/t, 16.06-7.07.2014; 1 ○', [SU1], $\mathrm{p} / \mathrm{t}, 23.06-4.09 .2014$.

RANGE. West Palaearctic boreo-nemoral.

NOTE. This is the northernmost record of the species.

Gnaphosa lapponum (L. Koch, 1866) *

MATERIAL. $8 \sigma^{7} \sigma^{7}, 2$ कᄋ, [Lo-lumt], p/t, 11-22.VI.2010; 1 $\sigma^{7}$, [K-lut3], p/t, 17-31.VII.2010; 3 우, [P-t] , p/t, 16-26.VI.2011.

RANGE. West Palaearctic boreo-montane.

Gnaphosa leporina (L. Koch, 1866) *

MATERIAL. $1 \mathrm{o}^{\top}$, [YU-3], p/t, 16.06-7.07.2014.

RANGE. Palaearctic boreal-nemoral.

Gnaphosa muscorum (L. Koch, 1866)

MATERIAL. 28 O $^{7}, 2$ 우, [K-ut2], p/t, 17-31.VII.2010; 25 $\sigma^{7} \sigma^{7}, 4$ 우, [K-lut3], p/t, 17-31.VII.2010; $7 \sigma^{7} \sigma^{7}$, [K-lut2], p/t, 17-31.VII.2010.

RANGE. Subcircum-Holarctic arcto-boreal.

Haplodrassus signifer (C.L. Koch, 1839)

MATERIAL. $4 O^{7} \sigma^{7}, 1$ \%, [Lo-be], p/t, 11-22.VI.2010; $10^{7}$, [Lo-lumt], p/t, 11-22.VI.2010.

RANGE. Holarctic polyzonal.

Haplodrassus soerenseni (Strand, 1900)

MATERIAL. $13 \bigcirc^{7} \sigma^{7}, 2$ 우, [Lo-be], p/t, 11-22.VI.2010; 2 $\sigma^{7} \sigma^{7}$, [Lo-lumt], p/t, 11-22.VI.2010; 10 O $^{7} \sigma^{2}, 3$ O+ , [P-p], p/t, 16-26.VI.2011; 8 O७ $^{\top}, 4$ क्ष, [P-b2], p/t, 16-26.VI.2011; 3 O $^{\top} \sigma^{\top}$, [P-b1], p/t, 16-26.VI.2011.

RANGE. Palaearctic boreo-nemoral.

Micaria aenea Thorell, 1871

MATERIAL. $90^{7} \sigma^{7}$, [YU-2] $, \mathrm{p} / \mathrm{t}, 16.06-7.07 .2014 ; 11 \sigma^{7} \sigma^{7}$, [YU-3], p/t, 16.06-7.07.2014.

RANGE. Holarctic boreo-nemoral.

NOTE. Species was recorded for the Kola Peninsula previously from Korablekk Mt., Pasvik Nature Reserve [Zenkova et al., 2013]. 
Micaria alpina L. Koch, 1872

MATERIAL. $1 \sigma^{7}$, [K-lt], p/t, 25-26.VI.2009; 1 , , [Lo-lumt], p/t, 11-22.VI.2010; $1 \sigma^{7}$, [VNE-4], sift., 26.VII.2011; $1 \sigma^{7}$, [YU2], p/t, 16.06-7.07.2014; $1 \mathrm{O}^{7}, 1$ q, [YU-3], p/t, 16.06-7.07.2014; $1 \bigcirc^{\prime}, 1$, [YUK-1], p/t, 27.06-30.09.2014.

RANGE. Holarctic boreo-montane.

Zelotes clivicola (L. Koch, 1870) *

MATERIAL. $1 \sigma^{7}$, [SU-2], p/t, 22.06-10.07.2013.

RANGE. West Palaearctic boreo-nemoral.

Zelotes subterraneus (C.L. Koch, 1833)

MATERIAL. $1 \bigcirc^{\text {7 }}$, [P-b2], p/t, 16-26.VI.2011; 1 , [YU-3],

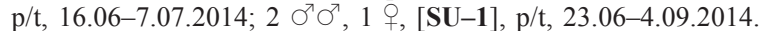

RANGE. European polyzonal.

Fam. HAHNIIDAE (3)

Cryphroeca silvicola (C.L. Koch, 1834)

PREVIOUS RECORDS. Vudyavrchorr Mt. [Kamayev, 2010; Tanasevitch, Kamayev, 2011]; near Kuelporr Vil. [Tanasevitch, Kamayev, 2011].

MATERIAL. $1 \sigma^{7}, 1$ \%, [V-pe1], s/s, VIII.2009; $1 \sigma^{7},[$ Vpe1], p/t, 6-16.VIII.2009; 1 О7, [VS-be], p/t, 6-16.VIII.2009; 2 $\mathcal{O}^{2} \mathcal{O}^{2},[\mathbf{V}-\mathbf{b s}], \mathrm{p} / \mathrm{t}, 6-16 . \mathrm{VIII} .2009 ; 1$ O, [VS-be], sift., h/c, VIII.2009; 1 \%, [V-bs], sift., h/c, VIII.2009; 1 + , [V-pe1], sift., VIII.2010; 4 우, [Bot-3], h/c, 22.VII.2011; 4 우, [Im-1] h/c, 30.VII.2011; $1 \mathrm{O}^{7}, 5$ Oᄋ+, [Bot-2], h/c, 22.VII.2011; $2 \mathrm{O}^{7} \mathrm{O}^{7}$, [Pp], p/t, 16-26.VI.2011.

RANGE. Palaearctic boreo-nemoral.

Hahnia ononidum Simon, 1875

PREVIOUS RECORDS. Vudyavrchorr Mt.; near Kuelporr Vil. [Tanasevitch, Kamayev, 2011].

MATERIAL. $1 \sigma^{7}, 2$ Oᄋ, [V-pe1], s/s, VIII.2009; $1 \sigma^{7}$, [VSbe], s/s, VIII.2009; 2 o $^{2} \sigma^{2}, 1$ \%, [V-bs], s/s, VIII.2009; 1 \%, [Vlut2], s/s, VIII.2010; $1 \mathrm{O}^{\top}, 1$ \%, [VNE-4], sift., 26.VII.2011.

RANGE. Holarctic boreo-nemoral.

Hahnia nava (Blackwall, 1841)

MATERIAL. $2 \mathrm{O}^{7} \sigma^{7}$, [SU-1], p/t, 23.06-4.09.2014.

RANGE. Palaearctic boreal-nemoral.

Fam. LINYPHIIDAE (102)

Abacoproeces saltuum (L. Koch, 1872)^

PREVIOUS RECORDS. Near Kuelporr Vil. [Tanasevitch, Kamayev, 2011].

RANGE. West Palaearctic boreo-nemoral.

Abiskoa abiskoensis (Holm, 1945) ^

PREVIOUS RECORDS. Near Kuelporr Vil. [Tanasevitch, Kamayev, 2011].

RANGE. Palaearctic boreal.

Agnyphantes expunctus (O. Pickard-Cambridge, 1875) MATERIAL. 1 \%, [V-pe1], h/c, VIII.2009.

RANGE. Palaearctic boreal.

Agyneta affinis (Kulczyński, 1898) *

MATERIAL. 5 O $^{7} \sigma^{7}$, [P-b2], p/t, 16-26.VI.2011.

RANGE. Palaearctic arcto-boreal.

NOTE. This is the northernmost record of the species.

Agyneta cauta (O. Pickard-Cambridge, 1902)

PREVIOUS RECORDS. Vudyavrchorr Mt. [Tanasevitch, Kamayev, 2011].
MATERIAL. $19 \bigcirc^{7} \odot^{7}, 5$ 우, [Lo-be], p/t, 11-22.VI.2010; 1 $\sigma^{7},[\mathbf{P}-\mathbf{t}], \mathrm{p} / \mathrm{t}, 16-26 . V I .2011 ; 19$ O$^{7} \sigma^{7}, 1$ + $,[\mathbf{P}-\mathbf{p}], \mathrm{p} / \mathrm{t}, 16-$ 26.VI.2011; 22 O $^{7} \sigma^{7}, 3$ ㅇ, [P-b2], p/t, 16-26.VI.2011.

RANGE. European boreo-nemoral.

Agyneta conigera (O. Pickard-Cambridge, 1863)

MATERIAL. $2 \sigma^{7} \sigma^{7}, 1$, [Lo-be], p/t, 11-22.VI.2010; $1 \sigma^{7}$,

[P-p], p/t, 16-26.VI.2011; 2 Oे $^{\mathbf{T}}$, [P-b1], p/t, 16-26.VI.2011.

RANGE. Palaearctic boreo-nemoral.

Agyneta gulosa (L. Koch, 1869)

MATERIAL. 2 O$^{7} \sigma^{7}$, [Lo-be], p/t, 11-22.VI.2010; 1 O $^{7}$, [Lolumt], s/s, 11.VI.2010; 1 , [K-lut3], p/t, 17-31.VII.2010; $1 \sigma^{7}$, [K-lut2], p/t, 17-31.VII.2010; 1 O $^{\text {T }, 1}$ +, [VN-1], h/c, 1.VIII.2011; 4 우, [Im-2], sift., 30.VII.2011; 1 o , 1 ㅇ, [Im-3], sift., h/c, 30.VII.2011; 2 우, $[\mathbf{I m}-\mathbf{2}], \mathrm{h} / \mathrm{c}$ under a stones on bald ground, 30.VII.2011; 1 +, [Bot-5], h/c, 22.VII.2011; 1 \% , [VN-2], h/c,

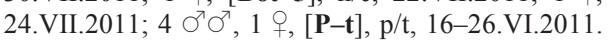

RANGE. Palaearctic boreo-nemoral.

Agyneta rurestris (C.L. Koch, 1836) *

MATERIAL. $4 \mathrm{O}^{\top} \sigma^{\top}, 2$ 우, [Lo-lumt], p/t, 11-22.VI.2010.

RANGE. West Palaearctic polyzonal.

NOTE. This is the northernmost record of the species.

Allomengea scopigera (Grube, 1859)

PREVIOUS RECORDS. Vudyavrchorr Mt. [Tanasevitch, Kamayev, 2011].

MATERIAL. 2 오, [VS-be], p/t, 6-16.VIII.2009; 1 , [VSbe], s/s, VIII.2010; 1 , [V-pe1], s/s, VIII.2010; 3 Oा $^{7}, 20$ 우, [VS-be], p/t, 7-17.VIII.2010; $2 \mathrm{O}^{7} \sigma^{7}$, [V-bs], p/t, 7-17.VIII.2010; $1 O^{7}, 1$ O, [V-ut2], p/t, 7-17.VIII.2010.

RANGE. Palaearctic-West Nearctic boreo-nemoral.

Bathyphantes eumenis (L. Koch, 1879) ^

PREVIOUS RECORDS. Vudyavrchorr Mt. [Tanasevitch, Kamayev, 2011].

RANGE. Holarctic boreo-nemoral.

Bathyphantes reprobus (Kulczyński, 1916) ${ }^{\wedge}$

PREVIOUS RECORDS. Near Kuelporr Vil. [Tanasevitch, Kamayev, 2011].

RANGE. Holarctic boreal. $1894^{\wedge}$

Bathyphantes setiger F.O. Pickard-Cambridge,

PREVIOUS RECORDS. Vicinity of the MSUFS [Tanasevitch, Kamayev, 2011].

RANGE. Palaearctic boreo-nemoral.

Bolephthyphantes index (Thorell, 1856)

PREVIOUS RECORDS. Vudyavrchorr Mt. [Tanasevitch,

Kamayev, 2011].

MATERIAL. $1 \sigma^{7},[$ [P-b2], p/t, 16-26.VI.2011.

RANGE. Palaearctic boreo-nemoral.

Bolyphantes alticeps (Sundevall, 1833)

MATERIAL. 1 ㅇ, [Bog], sw. of Chamerion sp. along bog edge, 1.VIII.2011.

RANGE. Palearctic boreo-nemoral.

NOTE. This is the northernmost record of the species.

Bolyphantes luteolus (Blackwall, 1833)

PREVIOUS RECORDS. Near Kuelporr Vil. [Tanasevitch, Kamayev, 2011].

MATERIAL. 1 \%, [VNE-4], sift., 26.VII.2011; 4 ㅇ, [B-1], sw., 1.VIII.2011; 1 \%, [VN-3], sift., 24.07.2011. 
RANGE. West Palaearctic boreo-nemoral.

Centromerus arcanus (O. Pickard-Cambridge, 1873) PREVIOUS RECORDS. Near Kuelporr Vil. [Tanasevitch, Kamayev, 2011]; Vudyavrchorr Mt. [Kamayev, 2010; Tanasevitch, Kamayev, 2011].

MATERIAL. $3 \sigma^{7} \sigma^{7}, 8$ क + , [V-ut1]; $13 \sigma^{7} \sigma^{7}$, [V-be2]; $17 \sigma^{7} \sigma^{7}$, [V-pe2]; $4 \bigcirc^{7} \sigma^{\top}, 14$ 우, [V-pe1], s/s, VIII.2009; 4 우, [VS-be], s/s, VIII.2009; 2 우, [V-bs], s/s, VIII.2009; 2 O $^{\top} \sigma^{2}, 13$ 우, [V-be1], sift., h/c, VIII.2009; 3 O $^{7} \sigma^{7}, 7$ 우, [V-ut2], s/s, VIII.2009; $1 \sigma^{7}, 19$ [VS-be], p/t, 6-16.VIII.2009; 3 우, [V-pe1], sift., h/c, VIII.2009; 3 $\sigma^{7} \sigma^{7}, 8$ O우, [V-bs], sift., h/c, VIII.2009; $3 \bigcirc^{7} \sigma^{7}, 8$ 우, [V-ut2], sift., h/c, VIII.2009; 1 + , [Lo-be], s/s, 14.IX.2009; 5 o $^{7} \sigma^{7}, 1$, , [Lo-be],

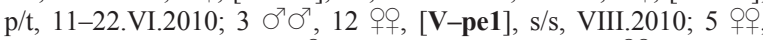
[VS-be], s/s, VIII.2010; 1 क, [V-bs], s/s, VIII.2010; 2 क्+, [V-be1], s/s, VIII.2010; 3 o $^{7} \sigma^{7}, 12$ 우, [V-ut2], s/s, VIII.2010; 3 ○ $^{7} \sigma^{7}, 7$ 우, [V-pe1], sift., VIII.2010; $10^{7}, 5$ 우, [VS-be], sift., VIII.2010; 8 우, [V-bs], sift., VIII.2010; 3 우, [V-be1], sift., VIII.2010; 3 O $^{7} \sigma^{7}, 21$ 우, [V-ut2], sift., VIII.2010; 1 + [VNE-2], sift., 25.VII.2011; $10^{7}$,

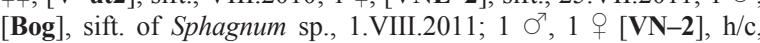
24.VII.2011; 3 O $^{7} \sigma^{7}$, [P-t] , p/t, 16-26.VI.2011; 18 O $^{7} \sigma^{7}, 1$ ㅇ, [P-p], p/t, 16-26.VI.2011; 2 O' $^{7}, 1$ +, [P-b2], p/t, 16-26.VI.2011; 99 $\mathrm{O}^{7} \mathrm{O}^{7}, 1$ 穴, [P-b1] $, \mathrm{p} / \mathrm{t}, 16-26 . \mathrm{VI} .2011$.

RANGE. European-Middle Siberian boreo-nemoral.

Centromerus incilium (L. Koch, 1881) ${ }^{\wedge}$

PREVIOUS RECORDS. Vudyavrchorr Mt. [Tanasevitch, Kamayev, 2011].

RANGE. West Palaearctic boreo-nemoral.

Centromerus sylvaticus (Blackwall, 1841)

PREVIOUS RECORDS. Vicinity of the MSUFS; near Kuelporr Vil.; Vudyavrchorr Mt. [Tanasevitch, Kamayev, 2011].

MATERIAL. $1 \sigma^{7}$, [VS-be], s/s, VIII.2009; 3 + , [V-bs], p/t, 6-16.VIII.2009; 1 O', 1 \% [VS-be], p/t, 6-16.VIII.2009; 1 +, [Vbs], sift., h/c VIII.2009; 1 o', [VS-be], s/s, VIII.2010; 1 , [V-bs], s/s, VIII.2010; 1 , , [V-be1], s/s, VIII.2010; 4 우, [VS-be], p/t, $7-$ 17.VIII.2010; 3 of , [V-bs], p/t, 7-17.VIII.2010; 1 O', [V-bs], sift., VIII.2010; 2 OO, [V-be1], p/t, 7-17.VIII.2010; 1 \%, [V-be1] sift., VIII.2010; 3 O $^{\prime} \sigma^{\prime}, 6$ OP, [Bot-1], sift., h/c, 22.VII.2011; 3 $\sigma^{7} \sigma^{3}, 1$,,$[\mathbf{V u}-3$ ], sift., 23.VII.2011; 1 +, [P-p], p/t, 16-26.VI.2011; 2 웅, [P-b1], p/t, 16-26.VI.2011.

RANGE. Holarctic boreo-nemoral.

Ceraticelus bulbosus (Emerton, 1882) ${ }^{\wedge}$

PREVIOUS RECORDS. Near Kuelporr Vil. [Tanasevitch, Kamayev, 2011].

RANGE. Holarctic boreal.

Ceratinella brevipes (Westring, 1851)

PREVIOUS RECORDS. Near Kuelporr Vil. [Tanasevitch, Kamayev, 2011].

MATERIAL. $20^{7} \sigma^{7}, 1$, [Lo-be], p/t, 11-22.VI.2010; 1 , [Lo-lumt], p/t, 11-22.VI.2010; 1 + , [Lo-be], s/s, 11.VI.2010; $\sigma^{\prime}$, [Lo-lumt], s/s, 11.VI.2010; 1 o', [P-p], p/t, 16-26.VI.2011.

RANGE. Palaearctic boreo-nemoral.

Ceratinella brevis (Wider, 1834) ${ }^{\wedge}$

PREVIOUS RECORDS. Near Kuelporr Vil. [Tanasevitch, Kamayev, 2011].

RANGE. Palaearctic boreo-nemoral.

Ceratinella wideri (Thorell, 1871) *

MATERIAL. 1 +, [P-b2], p/t, 16-26.VI.2011.

RANGE. Palaearctic boreal.
Cnephalocotes obscurus (Blackwall, 1834)

MATERIAL. $1 \sigma^{7}, 1$ \% , [V-be1], sift., h/c, VIII.2009; $5 \sigma^{7} \sigma^{7}, 3$ 우, [Lo-be], p/t, 11-22.VI.2010; $3 \mathrm{O}^{\top} \sigma^{7}, 11$ 우, [Lo-lumt], p/t, 1122.VI.2010; 1 ㅇ, [K-ut2], p/t, 17-31.VII.2010; 11 o $^{7} \sigma^{7}, 6$ 우, [K-

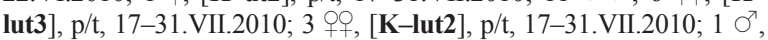

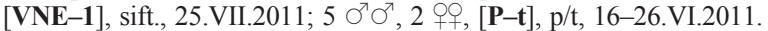
RANGE. Holarctic boreo-nemoral.

Decipiphantes decipiens (L. Koch, 1879)

PREVIOUS RECORDS. Near Kuelporr Vil. [Tanasevitch, Kamayev, 2011].

MATERIAL. 1 +, [V-be1], sift., h/c, VIII.2009; 1 +, [Lo-be], p/t, 11-22.VI.2010; 2 우, [Im-3], sift., h/c, 30.VII.2011; 2 o $^{7} \sigma^{\prime \prime}$, [P-t], p/t, 16-26.VI.2011.

RANGE. West Palaearctic boreal.

Dicymbium nigrum (Blackwall, 1834) *

MATERIAL. 1 +, [Bot-1], sift., h/c, 22.VII.2011.

RANGE. West Palearctic boreo-nemoral.

NOTE. This is the northernmost record of the species.

Diplocentria bidentata (Emerton, 1882)

PREVIOUS RECORDS. Vudyavrchorr Mt. [Tanasevitch, Kamayev, 2011].

MATERIAL. 1 \%, [V-be1], sift., h/c, VIII.2009.

RANGE. Holarctic boreo-nemoral.

Diplocentria rectangulata (Emerton, 1915)

PREVIOUS RECORDS. Vudyavrchorr Mt.; near Kuelporr Vil. [Tanasevitch, Kamayev, 2011].

MATERIAL. 1 , [V-ut2], s/s, VIII.2009; 2 Or $^{7}, 3$ 우, [Vut2], sift., h/c, VIII.2009; 1 o', [Lo-be], p/t, 11-22.VI.2010; 1 \%, [V-ut2], s/s, VIII.2010; 1 + , [V-ut2], sift., VIII.2010.

RANGE. Subcircum-Holarctic boreal.

Diplostyla concolor (Wider, 1834) ${ }^{\wedge}$

PREVIOUS RECORDS. Near Kuelporr Vil. [Tanasevitch, Kamayev, 2011].

RANGE. Subcircum-Holarctic boreo-nemoral.

NOTE. This is the northernmost record of the species.

Drapetisca socialis (Sundevall, 1833) *

MATERIAL. 3 우, [Im-1] h/c, 30.VII.2011; $1 \sigma^{7}$, [YUK-1], $\mathrm{p} / \mathrm{t}, 27.06-30.09 .2014$.

RANGE. Palearctic boreal-nemoral.

Drepanotylus borealis Holm, 1945

PREVIOUS RECORDS. Vicinity of the MSUFS [Tanasevitch, Kamayev, 2011].

MATERIAL. 6 क + , [Vu-1],h/c, 23.VII.2011; $1 \mathrm{O}^{7}, 1$ + , [Vu3], sift., 23.VII.2011.

RANGE. Palaearctic boreal.

Entelecara erythropus (Westring, 1851) *

MATERIAL. 1 +, [Im-2], h/c under a stones on bald ground, 30.VII.2011.

RANGE. Palearctic boreo-nemoral.

Erigone arctica maritima Kulczyński, 1902

MATERIAL. 3 우, [Vu-1] , h/c, 23.VII.2011.

RANGE. West European-Altaian disjunct, littoral.

Erigone atra Blackwall, 1833

MATERIAL. 1 , , [Lo-ad], s/s, 14.IX.2009; 1 \%, [Bot-1] sift., h/c, 22.VII.2011; 2 O $^{7}, 11$ Oᄋ, [Vu-1], h/c, 23.VII.2011; $1 \sigma^{7}, 1$ ㅇ, [Vu-3], sift., 23.VII.2011. 
RANGE. Holarctic polyzonal.

Erigone capra Simon, 1884

MATERIAL. 1 + , [Bog], sw. of Carex sp., 1.VIII.2011; $20^{7} 0^{7}$, 3 oᄋ, [Vu-1], h/c, 23.VII.2011; $1 \bigcirc^{7}$, [Vu-3], sift., 23.VII.2011.

RANGE. Palaearctic-West Nearctic boreo-nemoral.

COMMENTS. Following Locket [1964] and Buckle et al. [2001] I accept that E. capra and E. dentigera O. Pickard-Cambridge, 1874 are distinct species, while these species mentioned as synonyms in World Spider Catalog [2015].

Erigone dentipalpis (Wider, 1834)

MATERIAL. 34 O $^{7} \sigma^{7}, 39$ 우, [Nef-3], h/c, 28.VII.2011.

RANGE. Holarctic polyzonal.

Estrandia grandaeva (Keyserling, 1886)

PREVIOUS RECORDS. Vudyavrchorr Mt. [Tanasevitch, Kamayev, 2011].

MATERIAL. $1 \bigcirc^{7}$, [VS-be], p/t, 6-16.VIII.2009; $1 \odot^{7}, 3$ ㅇ,

[VNE-5], sw., 26.VII.2011.

RANGE. Holarctic boreal.

Gnathonarium dentatum (Wider, 1834) ^

PREVIOUS RECORDS. Vicinity of the MSUFS [Tanasevitch, Kamayev, 2011].

RANGE. Palaearctic polyzonal.

Gonatium rubens (Blackwall, 1833)

PREVIOUS RECORDS. Vudyavrchorr Mt. [Tanasevitch, Kamayev, 2011].

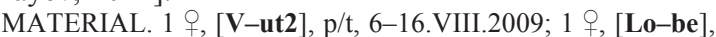
p/t, 11-22.VI.2010; 10 +, [Lo-lumt], p/t, 11-22.VI.2010; $1 \mathrm{O}^{\top}, 3$ 우, [K-lut3], p/t, 17-31.VII.2010; 3 우, [K-lut2], p/t, 1731.VII.2010; 2 우, [V-lut2], p/t, 7-17.VIII.2010; $10^{7}, 2$ 우, [Vlut2], sift., VIII.2010; $2 \mathrm{O}^{2} \mathrm{O}^{7}$, [Im-2], sift., 30.VII.2011; 1 \%, [Im3], sift., h/c, 30.VII.2011; 1 ㅇ, [VN-3], sift., 24.VII.2011; 3 우우, [VNE-1], sift., 25.VII.2011; 2 क्ष, [P-t], p/t, 16-26.VI.2011.

RANGE. Palaearctic polyzonal.

Hilaira herniosa (Thorell, 1875)

PREVIOUS RECORDS. Vudyavrchorr Mt. [Kamayev, 2010]; near Kuelporr Vil. [Tanasevitch, Kamayev, 2011].

MATERIAL. $2 \sigma^{7} \sigma^{7}, 3$ OP, [V-ut1]; $6 \sigma^{7} \sigma^{7}$, [V-be2]; $4 \sigma^{7} \sigma^{7}$, [V-pe2]; 1 O $^{7}, 1$, , [V-ut2], s/s, VIII.2009; 1 O $^{\top}, 9$ 우, [V-pe1], p/t, 6-16.VIII.2009; $1 \mathrm{O}^{\mathrm{V}}, 3$ 우, [VS-be], p/t, 6-16.VIII.2009; 4 우, [Vbs], p/t, 6-16.VIII.2009; 5 o' $0^{7}, 23$ OP, [V-ut2], p/t, 6-16.VIII.2009; 2 O+क, [V-bs], sift., h/c, VIII.2009; 1 + , [V-ut2], sift., h/c, VIII.2009; 1 o, [Lo-be], s/s, 14.IX.2009; 7 o' $0^{2}, 17$ OP, [Lo-be], p/t, 1122.VI.2010; 6 O $^{7} \sigma^{7}, 2$ + + , [Lo-lumt], p/t, 11-22.VI.2010; 6 O $^{7} \sigma^{7}, 2$ 우, [V-pe1], p/t, 7-17.VIII.2010; 1 \%, [V-be1], s/s, VIII.2010; 2 $O^{7} O^{7}, 3$ OO , [VS-be], p/t, 7-17.VIII.2010; $10^{7}, 5$ OO , [V-be1], p/t, 7-17.VIII.2010; 1 o', 4 우, [V-bs], p/t, 7-17.VIII.2010; 4 우, [Vut2], s/s, VIII.2010; 2 우, [V-be1], sift., VIII.2010; $5 \mathrm{O}^{7} \sigma^{7}, 13$ 우우, [V-ut2], p/t, 7-17.VIII.2010; $10^{7}, 1$ ㅇ, [V-ut2], sift., VIII.2010; 2 oq, [V-lut2], p/t, 7-17.VIII.2010; 1 q, [Im-3], sift., h/c, 30.VII.2011; 1 \%, [VNE-2], sift., 25.VII.2011; 3 qक , [VNE-4], sift., 26.VII.2011; $1 \sigma^{\prime}, 1$, [VN-3], sift., 24.VII.2011; 1 , [Nef-1], h/c, 28.VII.2011; 1 +, [VNE-1], sift., 25.VII.2011; $1 \sigma^{7}$, [P-t], p/t, 16-26.VI.2011; 1 $\sigma^{7}, 1$, [P-b2], p/t, 16-26.VI.2011; 2 o $^{7} \sigma^{\prime}, 1$ + $,[\mathbf{P}-\mathbf{b} 1], \mathrm{p} / \mathrm{t}, 16-$ 26.VI.2011.

RANGE. Holarctic boreal.

Hilaira nubigena Hull, 1911

MATERIAL. $1 \sigma^{7}$, [Bog], sift. of Sphagnum sp., 1.VIII.2011.

RANGE. Palaearctic-NW Nearctic boreal.

Horcotes strandi (Sytshevskaja, 1935)

PREVIOUS RECORDS. Vudyavrchorr Mt. [Tanasevitch, Kamayev, 2011].

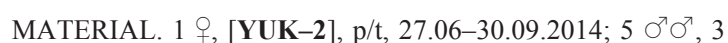
ㅇ, [YUK-3], p/t, 27.06-30.09.2014; 1 o,$[$ [YUK-3], s/s, 27.06.2014

RANGE. Palaearctic-NW Nearctic boreal.

Hypomma bituberculatum (Wider, 1834)

MATERIAL. 1 + , [Vu-1] , h/c, 23.VII.2011; 1 \% [Vu-3], sift., 23.VII.2011.

RANGE. Palaearctic polyzonal.

Hypselistes jacksoni (O. Pickard-Cambridge, 1902)

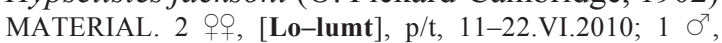
[VNE-4], sift., 26.VII.2011; 1 +, [Bog], sift. of Sphagnum sp., 1.VIII.2011.

RANGE. Palaearctic-West Nearctic boreo-nemoral.

Improphantes complicatus (Emerton, 1882) $\wedge$

PREVIOUS RECORDS. Near Kuelporr Vil. [Tanasevitch, Kamayev, 2011].

RANGE. Holarctic boreo-nemoral.

Incestophantes kochiellus (Strand, 1900) ^

PREVIOUS RECORDS. Near Kuelporr Vil. [Tanasevitch, Kamayev, 2011].

RANGE. Palaearctic boreal.

Kaestneria pullata (O. Pickard-Cambridge, 1863) MATERIAL. 1 , [B-1] , sw., 1.VIII.2011; $2 \bigcirc^{7} \sigma^{7}, 2$ 우, [Vu3], sift., 23.VII.2011.

RANGE. Holarctic polyzonal.

Leptorhoptrum robustum (Westring, 1851)

PREVIOUS RECORDS. Vicinity of the MSUFS [Tanasevitch, Kamayev, 2011]; Vudyavrchorr Mt. [Kamayev, 2010; Tanasevitch, Kamayev, 2011].

MATERIAL. 1 \%, [V-pe2]; 1 \%, [V-bs], s/s, VIII.2009; 13

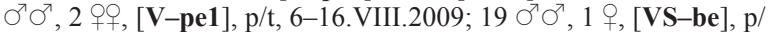
t, 6-16.VIII.2009; 34 O" $^{7}, 11$ 우, [V-bs], p/t, 6-16.VIII.2009; 1 $\mathrm{O}^{7}$, [V-ut2], p/t, 6-16.VIII.2009; 1 疋, [V-bs], sift., h/c, VIII.2009; $4 \sigma^{7} \sigma^{7}$, [V-pe1], s/s, VIII.2010; 2 oक , [VS-be], s/s, VIII.2010; 10 $\sigma^{7} \sigma^{7}, 6$ 우, [V-pe1], p/t, 7-17.VIII.2010; $12 \sigma^{7} \sigma^{7}, 7$ 우, [VS-be], p/t, 7-17.VIII.2010; 3 우, [VS-be], sift., VIII.2010; 14 O $^{7} 0^{7}, 2$ 우, [V-bs], p/t, 7-17.VIII.2010; 12 O $^{7}, 1$ +, [V-be1], p/t, 717.VIII.2010; $1 \sigma^{7}$, [V-ut2], p/t, 7-17.VIII.2010; 1 \%, [V-be1], sift., VIII.2010; 6 o $\sigma^{7}, 8$ OQ, [Bot-1], sift., h/c, 22.07.2011; $1 \sigma^{2}$, [Nef-3], h/c, 28.VII.2011; $1 \mathrm{O}^{7}, 1$ ㅇ, [Bot-5], h/c, 22.07.2011; 1 $\sigma^{7},[\mathbf{V u}-\mathbf{1}], \mathrm{h} / \mathrm{c}, 23$. VII.2011; 2 of, [Vu-3], sift., 23.VII.2011; 3

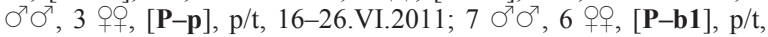
16-26.VI.2011.

RANGE. Palaearctic-West Nearctic boreo-nemoral.

Macrargus carpenteri (O. Pickard-Cambridge, 1894)

MATERIAL. 1 o , [P-t], p/t, 16-26.VI.2011.

RANGE. European boreal-nemoral.

NOTE. This is the northernmost record of the species.

Macrargus multesimus (O. Pickard-Cambridge, 1875)

MATERIAL. 1 + , [V-bs], sift., VIII.2009.

RANGE. Holarctic boreal.

Macrargus rufus (Wider, 1834)

PREVIOUS RECORDS. Near Kuelporr Vil.; Vudyavrchorr Mt. [Tanasevitch, Kamayev, 2011].

MATERIAL. 1 + , [VS-ut], s/s, 15.IX.2008; 1 $+,[\mathbf{V}-\mathbf{b s}], \mathrm{s} / \mathrm{s}$, VIII.2009; 1 \%, [V-pe1], p/t, 6-16.VIII.2009; 1 \%, [Lo-be], p/t, 11-22.VI.2010; 1 \%, [VN-3], sift., 24.VII.2011; 1 \% , [P-p], p/t, 16-26.VI.2011; 1 o', 1 +, [P-b1], p/t, 16-26.VI.2011. 
RANGE. European boreo-nemoral.

Maro minutus O. Pickard-Cambridge, 1906 PREVIOUS RECORDS. Vudyavrchorr Mt. [Tanasevitch, Kamayev, 2011].

MATERIAL. $1 \sigma^{7}$, [V-lut2], s/s, VIII.2009; $4 \sigma^{7} \sigma^{7},[\mathbf{P}-\mathbf{t}], \mathrm{p} / \mathrm{t}$, 16-26.VI.2011.

RANGE. European boreo-nemoral.

NOTE. This is the northernmost record of the species.

Maro sublestus Falconer, 1915

MATERIAL. $1 \sigma^{\top},[\mathbf{P}-\mathbf{t}], \mathrm{p} / \mathrm{t}, 16-26$. VI.2011.

RANGE. European boreal.

Maso sundevalli (Westring, 1851)

PREVIOUS RECORDS. Near Kuelporr Vil.; Vudyavrchorr Mt. [Tanasevitch, Kamayev, 2011].

MATERIAL. 1 \%, [V-be1], sift., VIII.2009; 1 +, [V-ut2], sift., VIII.2009; 1 O', [V-lut2], p/t, 6-16.VIII.2009; 1 + , [V-be1], s/s, VIII.2010; 1 +, [V-ut2], sift., VIII.2010; 2 우, [Im-3], sift., h/c, 30.VII.2011

RANGE. Holarctic polyzonal.

Mecynargus borealis (Jackson, 1930)

PREVIOUS RECORDS. Vudyavrchorr Mt. [Tanasevitch, Kamayev, 2011].

MATERIAL. 1 ㅇ, [V-lut1]; $3 \sigma^{7} \sigma^{\top}, 9$ 우, [V-lut2], s/s, VIII.2009; 2 우, [V-ut2], s/s, VIII.2009; $2 \sigma^{7} \sigma^{7}, 8$ 우, [V-ut2], s/ s, sift., VIII.2010; $1 \sigma^{T}$, [VN-3], sift., 24.VII.2011; $1 \sigma^{\top}, 7$ 우, [VNE-3], sift., 26.VII.2011; 1 +, [VNE-1], sift., 25.VII.2011.

RANGE. Subcircum-Holarctic boreal.

Mecynargus monticola (Holm, 1943)

PREVIOUS RECORDS. Near Kuelporr Vil.; Vudyavrchorr Mt. [Tanasevitch, Kamayev, 2011].

MATERIAL. 1 + [ [V-ut1]; $1 O^{\mathrm{T}}, 3$ $9+9,[\mathbf{V}-\mathbf{u t 2}$ ], s/s, VIII.2009; 5 우, [V-ut2], sift, h/c, VIII.2009; $2 \mathrm{O}^{\prime} \mathrm{O}^{\mathrm{T}}, 3$ Oᄋ, [V-ut2], s/s, VIII.2010; 5 Oㅇ, [V-ut2], sift., VIII.2010; 1 ㅇ, [VNE-2], sift., 25.VII.2011; 3 O' $\sigma^{7}, 2$ 우, [VNE-4], sift., 26.VII.2011.

RANGE. Palaearctic-West Nearctic boreal.

Mecynargus morulus (O. Pickard-Cambridge, 1873) PREVIOUS RECORDS. Vudyavrchorr Mt. [Tanasevitch, Kamayev, 2011].

MATERIAL. 1 \%, [V-pe1], sift., VIII.2009; $1 \bigcirc^{7}, 5$ 우, [Vut2], s/s, VIII.2009; 1 O', [V-lut2], s/s, VIII.2009; 2 के, [V-ut2], sift., h/c, VIII.2009; 1 + , [V-lut2], sift., h/c, VIII.2009; 1 O', [Lolumt], s/s, 14.IX.2009; 2 우, [Lo-be], s/s, 14.IX.2009; 1 +, [Lobe], sift., 11.VI.2010; $1 \mathrm{O}^{\text {T }}$, [K-lut2], p/t, 17-31.VII.2010; $1 \mathrm{O}^{\mathrm{T}}, 1$ o, [V-lut2], s/s, VIII.2010; 1 \%, [V-ut2], sift., VIII.2010; 9 ○ $^{7} \sigma^{7}$, 11 우, [Im-2], sift., 30.VII.2011; 3 Oొ $^{7}, 7$ 우, [Im-3], sift., h/c, 30.VII.2011; $3 \sigma^{7} \sigma^{7}, 3$ 우, [VNE-2], sift., 25.VII.2011; $1 \sigma^{7}, 1$, [VN-3], sift., 24.VII.2011; 1 O$^{7}, 4$ OO, [VNE-1], sift., 25.VII.2011; 2 of , [VNE-3], sift., 26.VII.2011; $2 \sigma^{7} \sigma^{7}, 2$ 우, [VN-2], h/c, 24.VII.2011; 1 O', 2 우, [P-t] ] p/t, 16-26.VI.2011.

RANGE. European boreo-nemoral. 1872)

Metopobactrus prominulus (O. Pickard-Cambridge,

MATERIAL. 1 O$^{7}$, [Lo-lumt], p/t, 11-22.VI.2010; 2 क्, [Lolumt], sift., 11.VI.2010.

RANGE. Holarctic polyzonal.

Micrargus herbigradus (Blackwall, 1854)

PREVIOUS RECORDS. Near Kuelporr Vil. [Tanasevitch, Kamayev, 2011].

MATERIAL. $1 \sigma^{7},\left[\right.$ V-pe1], sift., VIII.2009; $4 \sigma^{7} \sigma^{7},[\mathbf{L o}-$ lumt], p/t, 11-22.VI.2010; 1 +, [VNE-4], sift., 26.VII.2011; 3 $\sigma^{7} \sigma^{7},[\mathbf{P}-\mathbf{t}], \mathrm{p} / \mathrm{t}, 16-26$. VI.2011; $2 \mathrm{\sigma}^{7} \sigma^{7},[\mathbf{P}-\mathbf{b} 1], \mathrm{p} / \mathrm{t}, 16-26 . V I .2011$.
RANGE. Palaearctic boreo-nemoral.

Microneta viaria (Blackwall, 1841)

PREVIOUS RECORDS. Vicinity of MSUFS; near Kuelporr Vil. [Tanasevitch, Kamayev, 2011].

MATERIAL. $10^{7}$, [V-bs], sift., VIII.2009; $20 \sigma^{7} \sigma^{7}, 5$ 오, [Lobe], p/t, 11-22.VI.2010; 2 ㅇ, [Bot-1], sift., h/c, 22.VII.2011; 9 우,

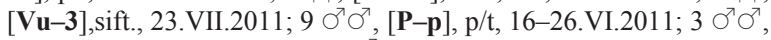
[P-b2], p/t, 16-26.VI.2011; 1 o', [P-b1], p/t, 16-26.VI.2011.

RANGE. Holarctic boreo-nemoral.

Minicia marginella (Wider, 1834)

MATERIAL. 1 ơ $^{7} 1$ \% , [YUK-1] ] p/t, 27.06-30.09.2014.

RANGE. Palaearctic boreal-nemoral.

Minyrioloides trifrons (O. Pickard-Cambridge, 1863)

MATERIAL. 1 + , [B-1], sw., 1.VIII.2011.

RANGE. Holarctic boreo-nemoral.

Minyriolus pusillus (Wider, 1834)

PREVIOUS RECORDS. Near Kuelporr Vil.; Vudyavrchorr Mt. [Tanasevitch, Kamayev, 2011].

MATERIAL. 1 +, [V-be1], sift., VIII.2009; $10^{\top}, 1$, [VN-2], h/c, 24.VII.2011; 1 o', [P-b1], p/t, 16-26.VI.2011.

RANGE. Palaearctic boreo-nemoral.

Moebelia penicillata (Westring, 1851) *

MATERIAL. 1 + [Im-2], sift., 30.VII.2011.

RANGE. European boreo-nemoral.

Neriene clathrata (Sundevall, 1830)

MATERIAL. $1 \sigma^{7}$, [P-b1], p/t, 16-26.VI.2011.

RANGE. Subcircum-Holarctic boreo-nemoral.

Neriene radiata (Walckenaer, 1842) ${ }^{\wedge}$

PREVIOUS RECORDS. Near Kuelporr Vil. [Tanasevitch, Kamayev, 2011].

RANGE. Subcircum-Holarctic boreo-nemoral.

NOTE. This is the northernmost record of the species.

Obscuriphantes obscurus (Blackwall, 1841)

MATERIAL. 1 \%, [B-1], sw., 1.VIII.2011; 1 \%, [Im-1], h/c, 30.VII.2011; 2 우, [VN-2], h/c, 24.VII.2011.

RANGE. West Palaearctic boreo-nemoral.

Oedothorax agrestis (Blackwall, 1853)

MATERIAL. 8 O $^{\top} \sigma^{7}, 29$ 우, [Nef-3], h/c, 28.VII.2011; $10^{\top}, 1$

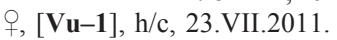

RANGE. West Palaearctic polyzonal.

Oedothorax apicatus (Blackwall, 1850) *

MATERIAL. 8 Ơ $^{7} \sigma^{7}, 3$ 우, [Nef-3], h/c, 28.VII.2011; 16 우, [Vu-1],h/c, 23.VII.2011.

RANGE. West Palaearctic polyzonal.

Oedothorax retusus (Westring, 1851)

MATERIAL. 1 O$^{\text {’ }, ~} 1$ + , [Vu-3], sift., 23.VII.2011.

RANGE. European-Middle Siberian polyzonal.

Oreoneta sinuosa (Tullgren, 1955)

MATERIAL. $60^{7} \sigma^{7}, 4$ oㅇ, [Lo-lumt], p/t, 11-22.VI.2010; 1 +, [Im-2], sift., 30.VII.2011; 1 \%, [Im-3], sift., h/c, 30.VII.2011; 1 $\sigma^{\prime}$, [VNE-2], sift., 25.VII.2011; $2 \sigma^{7} \sigma^{\prime}, 14$ के, [VNE-4], sift., 26.VII.2011; 4 O $^{7} \sigma^{7}, 9$ 우, [VN-3], sift., 24.07.2011; 1 ㅇ. [VNE3], sift., 26.VII.2011; 1 + $,[\mathbf{P}-\mathbf{t}], \mathrm{p} / \mathrm{t}, 16-26$. VI.2011.

RANGE. Fennoscandian boreal. 
NOTE. Previously only females of Oreoneta sp. were recorded in Khibiny (on Vudyavrchorr Mt.) [Tanasevitch, Kamayev, 2011].

Oreonetides vaginatus (Thorell, 1872)

PREVIOUS RECORDS. Vudyavrchorr Mt. [Tanasevitch, Kamayev, 2011].

MATERIAL. $3 \sigma^{7} \sigma^{7}$, [V-ut1]; $3 \sigma^{7} \sigma^{7}$, [V-be2]; $4 \sigma^{7} \sigma^{7}, 2$, 2 , [V-pe2]; 1 ○', [V-pe1], p/t, 6-16.VIII.2009; 1 \%, [VS-be], s/s, VIII.2009; 1 O , [V-bs], p/t, 6-16.VIII.2009; 1 क , [V-ut2], p/t, 616.VIII.2009; 1 O', [Lo-be], p/t, 11-22.VI.2010; $1 \sigma^{7}$, [Lo-lumt], p/t, 11-22.VI.2010; 2 O $^{7} \sigma^{7}, 1$ \%, [V-pe1], s/s, VIII.2010; 1 Oे $^{7},[\mathbf{V}-$ pe1], p/t, 7-17.VIII.2010; 1 9, [V-ut2], p/t, 7-17.VIII.2010; $10^{7}$, [V-pe1], sift.,VIII.2010; $11 \sigma^{7} \sigma^{7},[\mathbf{P}-\mathbf{t}], \mathrm{p} / \mathrm{t}, 16-26 . V I .2011 ; 5$ $\sigma^{7} \sigma^{7}, 2$ OP, [P-p], p/t, 16-26.VI.2011; $4 \bigcirc^{7} \sigma^{7},[\mathbf{P}-\mathbf{b} 2], \mathrm{p} / \mathrm{t}, 16-$ 26.VI.2011; 10 O $^{7} \mathbf{O}^{7},[\mathbf{P}-\mathbf{b} 1], \mathrm{p} / \mathrm{t}, 16-26 . V I .2011$.

RANGE. Holarctic arcto-boreal.

Oryphantes angulatus (O. Pickard-Cambridge, 1881) PREVIOUS RECORDS. Near Kuelporr Vil. [Tanasevitch, Kamayev, 2011].

MATERIAL. 1 + [VS-be], sift., h/c, VIII.2009; 1 , [VS-be], s/s, VIII.2010; 2 OO, [Vu-3], sift., 23.VII.2011; $1 \sigma^{7},[\mathbf{P}-\mathbf{t}]$, p/t, 16-26.VI.2011; 4 O $^{7} \mathrm{O}^{7}, 2$ 우, [P-p], p/t, 16-26.VI.2011; 6 O $^{7} \mathrm{O}^{2}$, [P-b1], p/t, 16-26.VI.2011.

RANGE. European boreo-nemoral.

Palliduphantes alutacius (Simon, 1884) ${ }^{\wedge}$

PREVIOUS RECORDS. Khibiny, near Kirovsk [Tanasevitch, 2008].

RANGE. West Palaearctic boreo-nemoral.

NOTE. This is the northernmost record of the species.

Palliduphantes antroniensis (Schenkel, 1933)

PREVIOUS RECORDS. Near Kuelporr Vil. [Tanasevitch, Kamayev, 2011]

MATERIAL. 1 \%, [V-ut2], p/t, 6-16.VIII.2009; $2 \sigma^{7} \sigma^{7}, 1$, [Lo-be], p/t, 11-22.VI.2010; $2 \sigma^{7} \sigma^{7}$, [Lo-lumt], p/t, 11-22.VI.2010 $1 \mathrm{O}^{\mathrm{T}}$, [Lo-be], sift., 11.VI.2010; $1 \mathrm{O}^{\mathrm{T}}$, [P-t], p/t, 16-26.VI.2011.

RANGE. European boreo-nemoral.

Panamomops mengei Simon, 1926

PREVIOUS RECORDS. Near Kuelporr Vil. [Tanasevitch, Kamayev, 2011].

MATERIAL. 1 \%, [V-pe1], sift., h/c, VIII.2009.

RANGE. West Palaearctic boreo-nemoral.

Pelecopsis mengei (Simon, 1884)

PREVIOUS RECORDS. Vudyavrchorr Mt.; near Kuelporr Vil. [Tanasevitch, Kamayev, 2011].

MATERIAL. 2 우, [V-pe2]; 10 , [V-bs], s/s, VIII.2009; 1 , [V-bs], p/t, 6-16.VIII.2009; $10^{7}, 1$, [V-bs], sift.h/c, VIII.2009; 1 , [Lo-be], p/t, 11-22.VI.2010; 3 O $^{7} 0^{7}, 11$ 우, [Lo-lumt], p/t, 11-22.VI.2010; 4 우, [V-bs], s/s, VIII.2010; 3 우요 [V-bs], sift.,

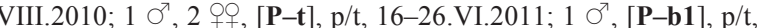
16-26.VI.2011.

RANGE. Holarctic boreo-nemoral

Pocadicnemis pumila (Blackwall, 1841)

MATERIAL. 2 \%O, [V-pe1], sift., h/c, VIII.2009; 2 O $^{7} \mathrm{O}^{7}$, [Lolumt], p/t, 11-22.VI.2010; 1 +, [Vu-3], sift., 23.VII.2011; $1 \sigma^{7}$ [P-t], p/t, 16-26.VI.2011; 1 ○', [P-p], p/t, 16-26.VI.2011.

RANGE. Holarctic boreo-nemoral

Poeciloneta variegata (Blackwall, 1841)

MATERIAL. 1 \%, [YU-2], p/t, 16.06-7.07.2014.

RANGE. Palaearctic-West Nearctic boreo-nemoral.
NOTE. This is the first record of species for the Kola Peninsula for the last 100 years. Previously it was recorded in 1910 in Vicinities of Murmansk Biological Station [Fedotov, 1912], Kola Inlet, Ekaterininskaya harbour.

Porrhomma campbelli F.O. Pickard-Cambridge, $1894 *$

MATERIAL. 1 ㅇ, [B-1] , sw., 1.VIII.2011.

RANGE. West Palaearctic boreo-nemoral.

Porrhomma egeria Simon, 1884

PREVIOUS RECORDS. Vudyavrchorr Mt. [Tanasevitch, Kamayev, 2011].

MATERIAL. $1 \bigcirc^{7},[\mathbf{V}-\mathbf{b s}]$, p/t, 6-16.VIII.2009; 1 ㅇ, [V-ut2], $\mathrm{p} / \mathrm{t}, 6-16$. VIII.2009.

RANGE. European boreo-nemoral.

Porrhomma pallidum Jackson, 1913

PREVIOUS RECORDS. Near Kuelporr Vil.; Vudyavrchorr Mt. [Tanasevitch, Kamayev, 2011].

MATERIAL. 1 \%, [V-bs], sift., VIII.2009; 1 \%, [Lo-be], p/t, 11-22.VI.2010; 1 o, [V-pe1], s/s, VIII.2010; 1 ․ [VS-be], s/s, VIII.2010; 1 , [V-pe1], sift., VIII.2010; 1 +, [V-ut2], sift., VIII.2010.

RANGE. Palaearctic polyzonal.

Porrhomma pygmaeum (Blackwall, 1834)

MATERIAL. 1 o', [Vu-4], h/c, 23.VII.2011.

RANGE. Palaearctic polyzonal.

Savignia frontata Blackwall, 1833

PREVIOUS RECORDS. Near Kuelporr Vil. [Tanasevitch, Kamayev, 2011].

MATERIAL. 1 , [V-pe1], sift., VIII.2009; $9 \sigma^{7} \sigma^{7}, 2$ ㅇ, [Nef-3], h/c, 28.VII.2011; 6 +क , [Vu-1], h/c, 23.VII.2011; 9 o' o', 11 우, [Vu-3],sift., 23.VII.2011.

RANGE. West Palaearctic boreo-nemoral.

Savignia producta Holm, 1977

MATERIAL. 1 + , [V-lut2], sift., VIII.2009; 1 O', [VNE-1], sift., 25.VII.2011

RANGE. West Palaearctic boreal.

Semljicola faustus (O. Pickard-Cambridge, 1900)

PREVIOUS RECORDS. Near Kuelporr Vil.; Vudyavrchorr Mt. [Tanasevitch, Kamayev, 2011].

MATERIAL. $1 \sigma^{\text {T }}$, [V-be2]; $19,[\mathbf{V}-\mathbf{p e 2}]$; $2 \circ \circ$, [VS-be], s/ s, VIII.2009; 1 or, [V-bs], s/s, VIII.2009; 2 우, [V-bs], p/t, 616.VIII.2009; 1 \%, [V-pe1], s/s, VIII.2010; $2 \sigma^{7} \sigma^{7}, 6$ OO , [VSbe], s/s, VIII.2010; 1 O [V-pe1], sift., VIII.2010; 1 ot, 6 오, [VS-be], sift., VIII.2010; 1 ㅇ, [V-bs], s/s, VIII.2010; 1 o $^{2}, 5$ 우, [V-be1], sift., VIII.2010; 1 O', 1 \%, [V-bs], sift., VIII.2010; 3 $\sigma^{\top} \sigma^{\top},[\mathbf{P}-\mathbf{b} 1], \mathrm{p} / \mathrm{t}, 16-26 . \mathrm{VI} .2011$.

RANGE. European boreo-nemoral.

Semljicola latus (Holm, 1939)

PREVIOUS RECORDS. Vudyavrchorr Mt.; near Kuelporr Vil. [Tanasevitch, Kamayev, 2011].

MATERIAL. 1 + , [V-ut1]; 3 O $^{7} \sigma^{7}, 1$, , [V-pe1], s/s, VIII.2009;

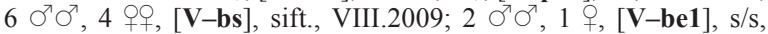
VIII.2009; $1 \mathrm{O}^{7}$, [V-ut2], s/s, VIII.2009; $1 \mathrm{O}^{\pi}, 1$ \%, [V-be1], sift., VIII.2009; 2 우, [Lo-be], p/t, 11-22.VI.2010; 1 ot, 1 오, [V-pe1], sift., VIII.2010.

RANGE. Palaearctic boreal

Sisicus apertus (Holm, 1939)

MATERIAL. 1 +, [V-pe1], sift., VIII.2009. 
RANGE. Holarctic boreo-nemoral.

Tallusia experta (O. Pickard-Cambridge, 1871) PREVIOUS RECORDS. Vudyavrchorr Mt. [Tanasevitch, Kamayev, 2011].

MATERIAL. 1 + , [V-bs], sift., VIII.2009.

RANGE. Palaearctic disjunct boreo-nemoral.

Tapinocyba pallens (O. Pickard-Cambridge, 1872) PREVIOUS RECORDS. Vudyavrchorr Mt.; near Kuelporr Vil. [Tanasevitch, Kamayev, 2011].

MATERIAL. $2 \sigma^{7} \sigma^{7}, 1$, [V-be2]; $4 \sigma^{\top} \sigma^{7}, 1$, [ [V-pe2]; 3 ㅇ, [V-bs], s/s, VIII.2009; 6 कᄋ, [V-be1], s/s, VIII.2009; 1 O , [Lobe], p/t, 11-22.VI.2010; 1 , [VS-be], s/s, VIII.2010; $2 \mathrm{O}^{7} \sigma^{7}, 7$ 우, [V-be1], s/s, VIII.2010; 6 우, [V-be1], sift., VIII.2010; $1 \mathrm{O}^{\text {T" }}$ 2 우, [VS-be], sift., VIII.2010; $1 \sigma^{7}, 1$ o, $[\mathbf{P}-\mathbf{p}], \mathrm{p} / \mathrm{t}, 16$ 26.VI.2011; 2 O $^{7}, 4$ 우, [P-b2], p/t, 16-26.VI.2011; $1 \sigma^{7},[\mathbf{P}-$ b1], p/t, 16-26.VI.2011.

RANGE. West Palaearctic boreo-nemoral.

Tenuiphantes alacris (Blackwall, 1853)

PREVIOUS RECORDS. Vudyavrchorr Mt.; near Kuelporr Vil. [Tanasevitch, Kamayev, 2011].

MATERIAL. 1 +,$[\mathbf{V}-\mathbf{u t 1}] ; 19,[\mathbf{V}-\mathbf{b e} 2] ; 1 \bigcirc^{7},[\mathbf{V}-\mathbf{p e 2}] ; 19$, [V-bs], p/t, 6-16.VIII.2009; 1 o', [V-be1], sift., VIII.2009; 1 , [V-bs], sift., VIII.2009; 3 क⿻ , [Lo-be], p/t, 11-22.VI.2010; $10^{7}$, [V-pe1], p/t, 7-17.VIII.2010; 2 우, [V-ut2], s/s, VIII.2010; 2 우, [V-be1], p/t, 7-17.VIII.2010; 2 q+, [V-ut2], p/t, 7-17.VIII.2010; 3 O$^{7} \sigma^{7}, 4$ 우, [P-p], p/t, 16-26.VI.2011; 3 Oक, [P-b2], p/t, 1626.VI.2011.

RANGE. Palaearctic boreo-nemoral.

Tenuiphantes mengei (Kulczyński, 1887)

MATERIAL. 1 , [Vu-3], sift., 23.VII.2011.

RANGE. Palaearctic boreo-nemoral.

Tenuiphantes nigriventris (L. Koch, 1879)

PREVIOUS RECORDS. Vudyavrchorr Mt. [Tanasevitch, Kamayev, 2011].

MATERIAL. 1 \%, [V-pe1], s/s, VIII.2009; $8 \sigma^{7} \sigma^{7}, 3$ 우, [Vbs], p/t, 6-16.VIII.2009; 5 O' $\sigma^{7}, 7$ 우, [V-pe1], p/t, 6-16.VIII.2009; 2 O+क, [V-bs], sift., VIII.2009; $1 \bigcirc^{7}, 3$ 우, [V-be1], sift., VIII.2009; 2 OO, [V-pe1], s/s, VIII.2010; $3 \mathrm{O}^{7} \sigma^{7}, 4$ OO, [VS-be], p/t, 717.VIII.2010; 1 ○", 1 \%, [V-pe1], p/t, 7-17.VIII.2010; 4 ○ $^{7}, 4$ 우, [V-bs], p/t, 7-17.VIII.2010; 4 ○' $^{7}, 2$ 우, [V-be1], p/t, 717.VIII.2010; 1 + , [VS-be], sift., VIII.2010; 1 + [V-be1], sift., VIII.2010; $1 \sigma^{7}$, [VNE-5], sw., 26.VII.2011; $2 \sigma^{7} \sigma^{7}, 3$ + , [Bot1], sift., h/c, 22.VII.2011.

RANGE. Palaearctic boreo-nemoral.

Tenuiphantes tenebricola (Wider, 1834)

PREVIOUS RECORDS. Vudyavrchorr Mt.; near Kuelporr Vil. [Tanasevitch, Kamayev, 2011].

MATERIAL. 1 , [V-ut1]; 1 + , [V-be2]; $2+9,[\mathbf{V}-\mathbf{p e 1}], \mathrm{s} / \mathrm{s}$, VIII.2009; $1 \sigma^{7}, 4$ 우, [VS-be], s/s, VIII.2009; $2 \sigma^{7} \sigma^{7}, 2$ 우, [VSbe], p/t, 6-16.VIII.2009; 1 O', 14 क्+, [V-pe1], p/t, 6-16.VIII.2009;

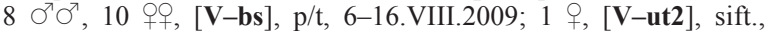
VIII.2009; 1 O $^{7}, 5$ 우, [V-be1], sift., VIII.2009; 3 O $^{7} \sigma^{7}, 2$ 우, [V-

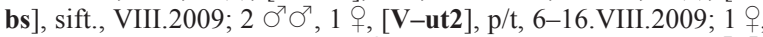
[Lo-be], p/t, 11-22.VI.2010; 1 + [ [V-be1], s/s, VIII.2010; 2 O $^{7} \sigma^{7}$, 5 우, [V-be1], p/t, 7-17.VIII.2010; 1 ㅇ, [V-pe1], p/t, 717.VIII.2010; 1 9, [VS-be], p/t, 7-17.VIII.2010; 3 of , [VS-be], sift., VIII.2010; 1 ; , [V-bs], p/t, 7-17.VIII.2010; 7 우, [V-ut2], p/ t, 7-17.VIII.2010; 1 \%, [VNE-2], sift., 25.VII.2011; 1 O $^{7}$, [Bot-1], sift., h/c, 22.VII.2011; 2 우, [Vu-3], sift., 23.VII.2011; 8 o $^{7} \sigma^{7}, 3$ 우, [P-p], p/t, 16-26.VI.2011; 1 + , [P-b2], p/t, 16-26.VI.2011; 7

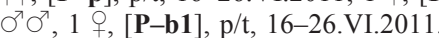

RANGE. West Palaearctic boreo-nemoral.
Tibioplus diversus (L. Koch, 1879)

PREVIOUS RECORDS. Near Kuelporr Vil.; Vudyavrchorr Mt. [Tanasevitch, Kamayev, 2011].

MATERIAL. $1 \sigma^{7}$, [Lo-be], p/t, 11-22.VI.2010; $1 \sigma^{7}, 2$ + 9 , [V-pe1], s/s, VIII.2009; 1 +, [V-pe1], s/s, VIII.2010

RANGE. Palaearctic-NW Nearctic boreal.

Tiso aestivus (L. Koch, 1872)

MATERIAL. 1 \%, [Lo-lumt], p/t, 11-22.VI.2010; 1 o', [YUK3], p/t, 27.06-30.09.2014.

RANGE. Holarctic boreal.

Walckenaeria antica (Wider, 1834)

MATERIAL. $1 \sigma^{7}$, [YU-2], p/t, 16.06-7.07.2014.

RANGE. European-Middle Siberian boreo-nemoral.

Walckenaeria capito (Westring, 1861)

PREVIOUS RECORDS. Vicinity of MSUFS [Tanasevitch, Kamayev, 2011].

MATERIAL. 1 +, [V-lut2], sift., VIII.2009; 2 우, [Lo-be], p/ t, 11-22.VI.2010; 1 \%, [V-lut2], s/s, VIII.2010; 1 + [ [VN-3], sift., 24.VII.2011; 1 ○', [YUK-3], p/t, 27.06-30.09.2014.

RANGE. Holarctic boreo-nemoral.

Walckenaeria cuspidata Blackwall, 1833

PREVIOUS RECORDS. Vicinity of MSUFS [Tanasevitch, Kamayev, 2011].

MATERIAL. 1 +, [V-pe1], sift., VIII.2009; 1 \% [Im-3], sift., h/c, 30.VII.2011; 1 O', [YU-1], p/t, 16.06-7.07.2014; 1 \%, [YU2], s/s, 16.06.2014.

RANGE. European - Middle Siberian boreo-nemoral.

Walckenaeria dysderoides (Wider, 1834)

MATERIAL. $110^{7} \sigma^{7}, 2$ 우, [Lo-be], p/t, 11-22.VI.2010; 1 ㅇ, [Lo-be], s/s, 11.VI.2010; 2 o $\sigma^{7},[\mathbf{P}-\mathbf{p}], \mathrm{p} / \mathrm{t}, 16-26 . \mathrm{VI} .2011 ; 5$ $\mathrm{O}^{\mathrm{T}} \sigma^{7}$, [P-b2], p/t, 16-26.VI.2011.

RANGE. Palaearctic boreo-nemoral.

NOTE. This is the northernmost record of the species. 1873)

Walckenaeria karpinskii (O. Pickard-Cambridge,

PREVIOUS RECORDS. Near Kuelporr Vil.; Vudyavrchorr Mt. [Tanasevitch, Kamayev, 2011].

MATERIAL. 1 , [V-ut1]; 1 ․, [V-lut2], s/s, VIII.2009; 1 , [V-ut2], sift., VIII.2009; 1 , [V-lut2], sift., VIII.2009; 4 o $^{7} \sigma^{7}, 2$ 우, [Lo-be], p/t, 11-22.VI.2010; $1 \sigma^{7}$, [Lo-lumt], p/t, 1122.VI.2010; 1 , [V-lut2], s/s, VIII.2010; 1 + , [V-lut2], sift.,

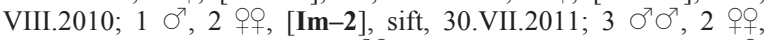
[VNE-4], sift., 26.VII.2011; 3 우, [VN-3], sift., 24.VII.2011; 1 , [VNE-1], sift., 25.VII.2011; 1 O', [VNE-3], sift., 26.VII.2011; 1 $\mathrm{O}^{7}, 1$ ㅇ, [VN-2], h/c, 24.VII.2011; 9 Ơ $^{\top} \sigma^{7},[\mathbf{P}-\mathbf{t}], \mathrm{p} / \mathrm{t}, 16-26$. VI.2011; $1 \mathrm{O}^{7},[\mathbf{P}-\mathbf{b} 1], \mathrm{p} / \mathrm{t}, 16-26 . \mathrm{VI} .2011$.

RANGE. Holarctic arcto-boreal.

Walckenaeria nudipalpis (Westring, 1851)

PREVIOUS RECORDS. Near Kuelporr Vil.; Vudyavrchorr Mt. [Tanasevitch, Kamayev, 2011].

MATERIAL. $1 \sigma^{7}$, [VS-be], sift., VIII.2009; 2 ㅇ, [V-be1], sift., VIII.2009; 1 \%, [V-lut2], sift., VIII.2009; 1 O$^{\text {T }}$ [VS-be], s/s, VIII.2010; 1 O", [Bog], sift. of Sphagnum sp., 1.VIII.2011.

RANGE. Palaearctic boreo-nemoral.

Zornella cultrigera (L. Koch, 1879)

PREVIOUS RECORDS. Vudyavrchorr Mt. [Tanasevitch,

Kamayev, 2011].

MATERIAL. $1 \sigma^{\text {r }}$, [V-pe2]; 1 \%, [V-ut1]; $1 \sigma^{\text {r }}$, [V-lut2], p/t, 6-16.VIII.2009; 18 우, [Lo-be], p/t, 11-22.VI.2010; 1 +, [V- 
be1], p/t, 7-17.VIII.2010; 1 +, [VNE-1], sift., 25.VII.2011; 3 $\sigma^{7} \sigma^{7}, 1$, [VNE-3], sift., 26.VII.2011; 1 \%, [VN-2], h/c, 24.VII.2011; 1 , , [P-t] $, \mathrm{p} / \mathrm{t}, 16-26 . V I .2011 ; 12$ +O , [P-p], p/t, 1626.VI.2011; 6 우, [P-b2], p/t, 16-26.VI.2011; 1 ㅇ, [P-b1], p/t, 16-26.VI.2011.

RANGE. Palaearctic boreal [Marusik et al., 2007; Tanasevitch, 2008].

\section{Fam. LIOCRANIDAE (2)}

Agroeca lusatica (L. Koch, 1875)*

MATERIAL. $1 \sigma^{\text {? }}$, [V-ut2], p/t, 6-16.VIII.2009.

RANGE. West Palaearctic boreo-nemoral.

NOTE. This is the northernmost record of the species.

Agroeca proxima (O. Pickard-Cambridge, 1871)

PREVIOUS RECORDS. Vudyavrchorr Mt. [Tanasevitch,

Kamayev, 2011]

MATERIAL. 1 +, [V-be1], h/c, VIII.2009; $1 \sigma^{\text {7 }}$, [V-ut2], p/t, 7-17.VIII.2010; 1 \%, [Vu-3], sift., 23.VII.2011.

RANGE. West Palaearctic boreal.

Fam. LYCOSIDAE (18)

Acantholycosa norvegica (Thorell, 1872)

PREVIOUS RECORDS. Vudyavrchorr Mt. [Tanasevitch, Kamayev, 2011].

MATERIAL. $1 \sigma^{7},[$ MSU], h/c, 27.VI.2009; 1 , [Lo-be], p/t, 11-22.VI.2010; 1 O' $^{7}$ [K-ut2], p/t, 17-31.VII.2010; 1 \%, [V-ut2], p/t, 7-17.VIII.2010; 1 + , [YU-1], p/t, 16.06-7.07.2014; $1 \sigma^{7}, 1$, [YU-2], p/t, 16.06-7.07.2014; $9 \mathrm{O}^{7} \mathrm{O}^{7}, 2$ 우, [YU-3], p/t, 16.067.07.2014; $4 \sigma^{7} \sigma^{7}, 1$, , [YUK-1], p/t, 27.06-30.09.2014.

RANGE. Palaearctic disjunct boreo-montane.

Alopecosa aculeata (Clerck, 1758)

MATERIAL. $4 \sigma^{7} \sigma^{7}$, [K-lt] ] p/t, 25-26.VI.2009; $1 \sigma^{7}, 3$ 우, [K-1], p/t, 25-26.VI.2009; 1 ऽ', [K-2], h/c, 26.VI.2009; 1 , [ [K-

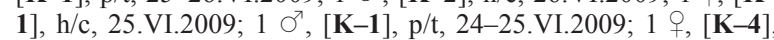

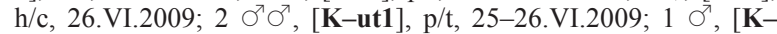
lut1], h/c, 25.VI.2009; 1 \%, [K-ut1], h/c, 26.VI.2009; 105 o' $0^{7}, 5$ 오, [Lo-be], p/t, 11-22.VI.2010; $3 \sigma^{7} \sigma^{7}$, [Lo-lumt], p/t, 11-

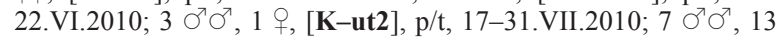
우, [K-lut3], p/t, 17-31.VII.2010; 1 क, [K-lut2 ], p/t, 1731.VII.2010; 1 , , [K-lut3], h/c, 17.VII.2010; 128 O $^{7} 0^{7}, 26$ 우, [Pt], p/t, 16-26.VI.2011; 1 O', [P-b2], p/t, 16-26.VI.2011.

RANGE. Holarctic boreo-nemoral.

Alopecosa pinetorum (Thorell, 1856) *

MATERIAL. $3 \sigma^{7} \sigma^{7}, 1$ \%, [Lo-be], p/t, 11-22.VI.2010; $1 \sigma^{7}$, [P-t], p/t, 16-26.VI.2011.

RANGE. European-Middle Siberian boreo-nemoral.

Alopecosa pulverulenta (Clerck, 1758)

PREVIOUS RECORDS. Vudyavrchorr Mt. [Tanasevitch,

Kamayev, 2011].

MATERIAL. 1 ,, [V-ut2], h/c, VIII.2009.

RANGE. Palaearctic boreo-nemoral.

Alopecosa taeniata (C.L. Koch, 1835) *

MATERIAL. $100^{7} \sigma^{7}, 3$ 우, [P-p], p/t, 16-26.VI.2011; 73 $\mathrm{O}^{7} \sigma^{7}, 13$ 우, [P-b2], p/t, 16-26.VI.2011; 1 o $^{7}, 1$ + $,[\mathbf{P}-\mathbf{b} 1], \mathrm{p} / \mathrm{t}$, 16-26.VI.2011.

RANGE. Palaearctic boreo-nemoral.

Arctosa alpigena (Doleschall, 1852)

PREVIOUS RECORDS. Vudyavrchorr Mt. [Tanasevitch, Kamayev, 2011].
MATERIAL. 2 우, [V-lut2], p/t, 6-16.VIII.2009; $10^{\text {T] }, ~[L o-~}$ lumt], p/t, 11-22.VI.2010; $1 \sigma^{7}, 5$ क⿻ , [K-ut2], p/t, 17-31.VII.2010; 1 , [V-lut2], p/t, 7-17.VIII.2010; 1 , [VN-1], h/c, 1.VIII.2011; 5 o $\sigma^{7}, 2$ 우, [P-t], p/t, 16-26.VI.2011.

RANGE. Holarctic arcto-boreal.

Pardosa agricola (Thorell, 1856)

MATERIAL. $3 \sigma^{7} \sigma^{7}$, [SU-1], p/t, 23.06-4.09.2014.

RANGE. European-Middle Siberian boreo-nemoral.

Pardosa amentata (Clerck, 1758)

PREVIOUS RECORDS. Vudyavrchorr Mt. [Tanasevitch, Kamayev, 2011].

MATERIAL. 1 , forest near the Kamennoe lake, h/c, 5.VI.2008; 2 o $^{7} \sigma^{7},\left[\right.$ K-be], p/t, 25-26.VI.2009; $1 \sigma^{7},[\mathbf{V L}], \mathrm{p} / \mathrm{t}$, 27-28.VI.2009; 1 O', 2 우, [V-be1], h/c, VIII.2009; 4 우, [Nef3], h/c, 28.VII.2011; 3 우, [Bot-4], h/c, 22.VII.2011; 1 +, [Bot-1], sift., h/c, 22.VII.2011; 2 우, [Vu-1], h/c, 23.VII.2011; 1 + , [VN1], h/c, 1.VIII.2011; 1 + , [P-t], p/t, 16-26.VI.2011.

RANGE. European boreo-nemoral.

Pardosa eiseni (Thorell, 1875)

PREVIOUS RECORDS. Vudyavrchorr Mt. [Tanasevitch, Kamayev, 2011].

MATERIAL. $2 \sigma^{7} \sigma^{7}$, [K-lt], p/t, 25-26.VI.2009; 3 o $^{7} \sigma^{7}, 1$, [K-2], h/c, 26.VI.2009; 1 O', 1 9, [K-ut1], p/t, 25-26.VI.2009; 2 $\mathrm{O}^{\mathrm{T}} \sigma^{7}, 1$, + , [K-1], h/c, 25.VI.2009; $1 \mathrm{O}^{\mathrm{T}}, 2$ 우, [K-4], h/c, 26.VI.2009;

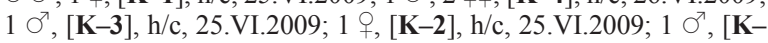
It], p/t, 25-26.VI.2009; 3 우, [V-ut2], p/t, 6-16.VIII.2009; 8 우, [V-lut2], p/t, 6-16.VIII.2009; 303 O' $^{7}, 58$ 우, [Lo-be], p/t, 1122.VI.2010; 5 O $^{7}, 1$ \%, [Lo-lumt], p/t, 11-22.VI.2010; 1 \%, [Lolumt], sift., 11. VI.2010; 2 우, [K-lut3], p/t, 17-31.VII.2010; 7 우, [K-lut2], p/t, 17-31.VII.2010; 5 우, [V-lut2], p/t, 7-17.VIII.2010; 1 㝏, [V-ut2], p/t, 7-17.VIII.2010; 1 \%, [V-lut2], h/c, VIII.2010; 1 \%, [Im-3], sift., h/c, 30.VII..2011; $68 \mathrm{O}^{7} \mathrm{O}^{\top}, 13$ 우, [P-t], p/t, 1626.VI.2011; 1 o', [P-b2], p/t, 16-26.VI.2011.

RANGE. Palaearctic arcto-boreal.

Pardosa hyperborea (Thorell, 1872)

PREVIOUS RECORDS. Vudyavrchorr Mt. [Tanasevitch, Kamayev, 2011].

MATERIAL. $1 \sigma^{\text {T }}$, [V-ut1]; 1 ㅇ, [K-lut1], h/c, 25.VI.2009; 1 +, [V-ut2], p/t, 6-16.VIII.2009; 4 우, [V-lut2], p/t, 6-16.VIII.2009; $49 \circlearrowleft^{7} \sigma^{7}, 7$ 우, [Lo-be], p/t, 11-22.VI.2010; 710 O $^{7} \sigma^{7}, 136$ 우웅, [Lo-lumt], p/t, 11-22.VI.2010; 2 क्+, [K-ut2], p/t, 17-31.VII.2010; 2 ㅇ, [V-ut2], p/t, 7-17.VIII.2010; 1 + , [V-lut2], sift., VIII.2010; 1 o. Kukisvumchorr Mt., dwarf shrub tundra, on the tent, $\mathrm{h} / \mathrm{c}$, 31.VII.2010; 1 \%, [Im-2], sift., 30.VII.2011; 1 , [VN-3], sift., 24.VII.2011; $47 \bigcirc^{7} \sigma^{7}, 14$ 우, [P-t], p/t, 16-26.VI.2011; $1 \bigcirc^{7},[\mathbf{P}-$ b2], p/t, 16-26.VI.2011.

RANGE. Subcircum-Holarctic boreo-montane.

Pardosa lapponica (Thorell, 1872)

MATERIAL. $3 \sigma^{7} \sigma^{7},[\mathbf{Y U}-2], \mathrm{p} / \mathrm{t}, 16.06-7.07 .2014 ; 1 \sigma^{7}$, [YU-3], p/t, 16.06-7.07.2014.

RANGE. Holarctic boreal.

Pardosa lugubris (Walckenaer, 1802)

MATERIAL. $1 \sigma^{7}$, [Lo-be], p/t, 11-22.VI.2010; 1 , [V-ut2], $\mathrm{h} / \mathrm{c}$, VIII.2009; 6 우, [Bot-4], h/c, 22.VII.2011; 3 o $^{7} \sigma^{7}, 1$ \% , [P-t], p/t, 16-26.VI.2011; 13 O O $^{7}, 1$ \%, [P-p], p/t, 16-26.VI.2011; 192 $0^{7} \sigma^{7}, 28$ 우, [P-b2], p/t, 16-26.VI.2011.

RANGE. Palaearctic boreo-nemoral.

Pardosa palustris (Linnaeus, 1758)

PREVIOUS RECORDS. Vudyavrchorr Mt. [Vasilyeva, Nekhaev, 2009].

MATERIAL. $5 \sigma^{7} \sigma^{7}, 4$ + + , [V], p/t, 11-12.VII.2008; $47 \sigma^{7} \sigma^{7}$, 
6 우, [Lo-be], p/t, 11-22.VI.2010; $7 \bigcirc^{7} \odot^{7}$, [Lo-lumt], p/t, 1122.VI.2010; 1 , [VN-1], h/c, 24.VII.2011; 1 , [VN-1], h/c, 1.VIII.2011; 4 O $^{7} \sigma^{7},[\mathbf{P}-\mathbf{t}], \mathbf{p} / \mathbf{t}, 16-26 . V I .2011 ; 1 \sigma^{7},[\mathbf{P}-\mathbf{b} 1], \mathbf{p} / \mathbf{t}$, 16-26.VI.2011.

RANGE. Palaearctic-West Nearctic boreo-ne-moral.

Pardosa prativaga (L. Koch, 1870) ${ }^{\wedge}$

PREVIOUS RECORDS. Vudyavrchorr Mt. [Tanasevitch, Kamayev, 2011].

RANGE. West Palaearctic boreo-nemoral.

Pardosa sphagnicola (F. Dahl, 1908)

PREVIOUS RECORDS. Vudyavrchorr Mt. [Tanasevitch, Kamayev, 2011].

MATERIAL. 1 \%, [V-bs], h/c, VIII.2009; $1 O^{\top 7},[$ Lo-lumt], p/t, 11-22.VI.2010.

RANGE. West Palaearctic boreo-nemoral.

Pirata piraticus (Clerck, 1758)

MATERIAL. $1 \mathrm{O}^{7}$, [SU-2], p/t, 22/06-10.07.2013.

RANGE. Holarctic polyzonal.

Trochosa terricola Thorell, 1856

MATERIAL. $2 \sigma^{7} \sigma^{7}, 1$, $,[\mathbf{P}-\mathbf{p}], \mathrm{p} / \mathrm{t}, 16-26 . V I .2011 ; 1+$, [Pb2], p/t, 16-26.VI.2011.

RANGE. Holarctic boreo-nemoral.

Xerolycosa nemoralis (Westring, 1861)

MATERIAL. $1 \sigma^{7}$, [Lo-be], p/t, 11-22.VI.2010; $1 \sigma^{7}$, [VN-

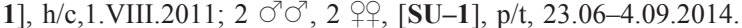

RANGE. Palaearctic boreo-nemoral.

Fam. MIMETIDAE (1)

Ero $\mathrm{sp}$.

MATERIAL. 1 juv, [YU-1], p/t, 16.06-7.07.2014.

Fam. MITURGIDAE (1)

Zora nemoralis (Blackwall, 1861)

MATERIAL. $1 \sigma^{7}$, [SU-1], p/t, 23.06-4.09.2014.

RANGE. Palaearctic boreo-nemoral.

Fam. PHILODROMIDAE (3)

Philodromus cespitum (Walckenaer, 1802) *

MATERIAL. 1 o, [Im-1],h/c, 30.VII.2011.

RANGE. Holarctic polyzonal.

Thanatus formicinus (Clerck, 1758)

MATERIAL. $2 \sigma^{7} \sigma^{7}$, [YUK-1], p/t, 27.06-30.09.2014; $1 \sigma^{7}, 1$

+ , [YUK-2], p/t, 27.06-30.09.2014.

RANGE. Holarctic boreo-nemoral.

Tibellus oblongus (Walckenaer, 1802)

MATERIAL. 1 +, [V-pe1], sift., h/c, VIII.2009; 1 \%, [V-pe1], s/s, VIII.2010.

RANGE. Holarctic boreo-nemoral.

Fam. SALTICIDAE (3)

Evarcha falcata (Clerck, 1758)

MATERIAL. 2 क, [V-pe1], sift., h/c, VIII.2009; $1 \sigma^{7}$, [YU-

3], p/t, 16.06-7.07.2014

RANGE. West Palaearctic boreo-nemoral.
Sitticus ranieri (Peckham et Peckham, 1909)

MATERIAL. $1 \sigma^{7}$, [Lo-lumt], p/t, 11-22.VI.2010; $1 \sigma^{7}$, [VN1], h/c, 1.VIII.2011; $1 \mathrm{O}^{7}$, [YU-3], p/t, 16.06-7.07.2014.

RANGE. Holarctic boreo-montane.

NOTE. Previously species was recorded for the Kola Peninsula from vicinity of the Monchegorsk city [Kronestedt, Logunov, 2001], but this finding was missed by Tanasevitch, Kamayev [2011].

Talavera aequipes (O. Pickard-Cambridge, 1871)

MATERIAL. 1 \%, [V-lut2], sift., VIII.2009; 1 \%, [V-lut2], s/s, VIII.2010.

RANGE. Palaearctic boreo-nemoral.

NOTE. This is the northernmost record of the species.

\section{Fam. TETRAGNATHIDAE (1)}

Tetragnatha extensa (Linnaeus, 1758)

MATERIAL. $1 \sigma^{\top}$, [Vu-2], sw., 23.VII.2011; 1 +, [B-2], sw., 1.VIII.2011.

RANGE. Holarctic polyzonal.

Fam. THERIDIIDAE (4)

Asagena phalerata (Panzer, 1801)

MATERIAL. $1 \mathrm{O}^{7}$, [YU-2], p/t, 16.06-7.07.2014.

RANGE. Palaearctic boreo-nemoral.

Robertus lividus (Blackwall, 1836)

PREVIOUS RECORDS. Vicinity of MSUFS; Vudyavrchorr Mt.; near Kuelporr Vil. [Tanasevitch, Kamayev, 2011].

MATERIAL. 1 , [V-ut1]; $1 \bigcirc^{7}, 2$ 우, [V-pe2]; $19,[\mathbf{K}-\mathbf{b e}]$, under a $\log , \mathrm{h} / \mathrm{c}, 25 . \mathrm{VI} .2009 ; 1 \sigma^{7}, 2$ 우, [V-pe1], s/s, VIII.2009; 1 $\mathrm{O}^{7}$, [VS-be], s/s, VIII.2009; 1 O', 1 ㅇ, [V-bs], p/t, VIII.2009; 4 $\sigma^{7} \sigma^{7}, 1$ 9, [V-be1], s/s, VIII.2009; 1 ○', [VS-be], p/t, 616.VIII.2009; 3 우, [V-be1], sift., VIII.2009; $1 \bigcirc^{\top}, 1$ +, [Lo-be], p/t, 11-22.VI.2010; 5 O $\sigma^{7}, 1$, [Lo-lumt], p/t, 11-22.VI.2010; 1 ot, [VS-be], s/s, VIII.2010; 2 o+ , [V-be1], s/s, VIII.2010; 1 O', 1 +, [VS-be], p/t, 7-17.VIII.2010; 1 o', [V-be1], p/t, 7-17.VIII.2010; 1 , [V-be1], sift., VIII.2010; 1 +, [V-ut2], sift., VIII.2010; 1 ;, [Nef-3], h/c, 28.VII.2011; 1 , [VNE-2], sift.., 25.VII.2011; 1 , $[\mathbf{I m}-2], \mathrm{h} / \mathrm{c}$ under a stones on bald ground, 30.VII.2011; 1 ; [VNE-4], sift., 26.VII.2011; 1 + , [Bot-1], sift., h/c, 22.VII.2011; 1 + , [VN-2], h/c, 24.VII.2011; $1 \mathrm{O}^{7}, 2$ 우, [Vu-3], sift., 23.VII.2011; $1 \mathrm{O}^{7}, 1$ ㅇ, [P-t ], p/t, 16-26.VI.2011; 2 O $^{7} \mathrm{O}^{7}, 3$ 우, [P-p], p/t, 16-

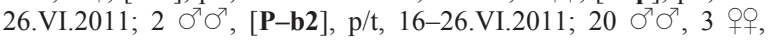
[P-b1], p/t, 16-26.VI.2011.

RANGE. Palaearctic-NW Nearctic boreo-nemoral.

Robertus lyrifer Holm, 1939

PREVIOUS RECORDS. near Kuelporr Vil. [Tanasevitch, Kamayev, 2011].

MATERIAL. 1 \%, [V-pe1], sift., VIII.2009.

RANGE. Holarctic boreal.

Robertus scoticus Jackson, 1914

PREVIOUS RECORDS. Vudyavrchorr Mt.; near Kuelporr Vil. [Tanasevitch, Kamayev, 2011].

MATERIAL. 3 우, [V-pe1], s/s, VIII.2009; 4 Or $^{7}, 17$ 우, [VS-be], s/s, VIII.2009; 3 ఠ $^{7}, 8$ 우, [VS-be], s/s, VIII.2010; 1 , [V-bs], s/s, VIII.2010; 3 O $^{7} \sigma^{7}, 1$ \%, [VS-be], p/t, 7-17.VIII.2010; 1

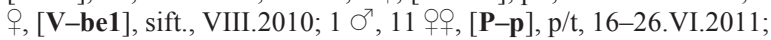
1 O$^{7},[\mathbf{P}-\mathbf{b} 2], \mathrm{p} / \mathrm{t}, 16-26 . V I .2011 ; 1 \sigma^{7},[\mathbf{P}-\mathbf{b} 1], \mathrm{p} / \mathrm{t}, 16-26 . V I .2011$. RANGE. European-Middle Siberian boreo-nemoral. 


\section{Fam. THOMISIDAE (7)}

Ozyptila arctica Kulczyński, 1908

PREVIOUS RECORDS. Vudyavrchorr Mt. [Kamayev, 2010; Tanasevitch, Kamayev, 2011].

MATERIAL. 1 ․ [V-ut2], p/t, 6-16.VIII.2009; 19 , [V-lut2], p/t, 6-16.VIII.2009; 1 O', [Lo-be], p/t, 11-22.VI.2010; 18 O O$^{7} \sigma^{7}, 2$ 오, [Lo-lumt], p/t, 11-22.VI.2010; 1 \%, [Lo-lumt], s/s, 11.VI.2010; 2 우, [K-ut2], p/t, 17-31.VII.2010; 1 , [V-lut2], sift., VIII.2010; $4 O^{7} \bigcirc^{7}, 1$, [ [Im-3], sift., h/c, 30.VII.2011; 3 +O , [VNE-4], sift., 26.VII.2011; $1 \sigma^{7}, 1$ \%, [VN-3], sift., 24.VII.2011; $1 \sigma^{7}, 1$ o,

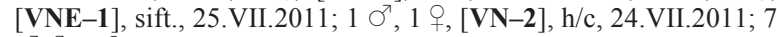
$\sigma^{7} \sigma^{7}, 1$ P, [P-t] $, \mathrm{p} / \mathrm{t}, 16-26$. VI.2011.

RANGE. Palaearctic-NW Nearctic arcto-boreal.

Ozyptila atomaria (Panzer, 1801)

MATERIAL. $1 \sigma^{7}, 1$, [YU-1] $, \mathrm{p} / \mathrm{t}, 27.06-30.09 .2014 ; 1 \sigma^{7}$, 1 ,,$[$ SU-1], p/t, 23.06-4.09.2014.

RANGE. Palaearctic boreo-nemoral.

Ozyptila trux (Blackwall, 1846)

MATERIAL. 1 +, [V-pe1], sift., VIII.2009; $1 \mathrm{O}^{\mathrm{T}},[\mathbf{P}-\mathbf{p}], \mathrm{p} / \mathrm{t}$, 16-26.VI.2011; 1 o', [P-b2], p/t, 16-26.VI.2011.

RANGE. Palaearctic boreo-nemoral.

Xysticus audax (Schrank, 1803)

MATERIAL. 1 O', [P-p], p/t, 16-26.VI.2011.

RANGE. Palaearctic boreo-nemoral.

Xysticus luctuosus (Blackwall, 1836)

PREVIOUS RECORDS. Vudyavrchorr Mt. [Tanasevitch, Kamayev, 2011]

MATERIAL. 1 + , [V-pe1], sift., h/c, VIII.2009; 8 O $^{7} \sigma^{7},[$ Lobe], p/t, 11-22.VI.2010.

RANGE. Holarctic boreo-nemoral.

Xysticus obscurus Collett, 1877

MATERIAL. 1 \%, [V-ut1]; 1 \% , [Lo-be], p/t, 11-22.VI.2010; $4 \sigma^{7} \sigma^{7}$, [Lo-lumt], p/t, 11-22.VI.2010; $11 \sigma^{7} \sigma^{7},[\mathbf{P}-\mathbf{t}], \mathrm{p} / \mathrm{t}, 16$ 26.VI.2011; 2 O $^{7} \sigma^{2}$, [P-b2], p/t, 16-26.VI.2011; 1 Oे $^{7}$ [P-b1], p/t, 16-26.VI.2011.

RANGE. Holarctic boreal.

Xysticus sabulosus (Hahn, 1832) ^

PREVIOUS RECORDS. Vicinity of MSUFS [Tanasevitch, Kamayev, 2011].

RANGE. European boreo-nemoral.

NOTE. This is the northernmost record of the species.

Fam. TITANOECIDAE (1)

Titanoeca nivalis Simon, $1874 *$

MATERIAL. $2 \sigma^{7} \sigma^{7}$, [YUK-1] ] p/t, 27.06-30.09.2014.

RANGE. Palaearctic-West Nearctic boreo-montane.

\section{Discussion}

Over 4800 adult spider specimens belonging to 145 species of 17 families were collected during our investigation. A total list of the species of Khibiny Mts consists of 161 species belonging to 17 families. The family Mimetidae was represented only by juvenile specimens. Thirty one species (see Table 1; including five species - Araniella proxima, Gnaphosa bicolor,
G. leporina, Zelotes clivicola, Titanoeca nivalis that were discovered during the final stage of this study) have not yet been found in other parts of the Kola Peninsula. Twenty species (Araniella proxima, Cyclosa conica, Gnaphosa bicolor, G. lapponum, G. leporina, Zelotes clivicola, Agyneta affinis, A. rurestris, Ceratinella wideri, Dicymbium nigrum, Drapetisca socialis, Entelecara erythropus, Moebelia penicillata, Oedothorax apicatus, Porrhomma campbelli, Agroeca lusatica, Alopecosa pinetorum, A. taeniata, Philodromus cespitum, Titanoeca nivalis) and one family (Titanoecidae) have been reported from the Kola Peninsula for the first time.

The family Linyphiidae accounts for $63 \%$ of the total species diversity and presents in all the habitats studied. Lycosidae amounts only for $11 \%$ of the total species diversity. The proportions of other families are insignificant and do not exceed 3\%, while Gnaphosidae and Thomisidae account for $6 \%$ and $4 \%$ respectively. The predominance of the linyphiids and lycosids is also a characteristic feature of the spider fauna of the entire Kola Peninsula [Tanasevitch, Kamayev, 2011], as well as of its tundra zone [Tanasevitch, Rybalov, 2010], and seems to be typical of European high altitude spider faunas [Koponen, 1993].

Some $90 \%$ of the species found in the Khibiny Mts display wide ranges, with the Holarctic and Palaearctic distribution being especially common. Boreo-nemoral and boreal species predominate and amount for $61 \%$ and $17 \%$ respectively. Arcto-boreal species are not numerous and account for 5\% only.

The most abundant species in the studied material are Agyneta cauta, Alopecosa aculeata, A. taeniata, Centromerus arcanus, Erigone dentipalpis, Gnaphosa muscorum, Hilaira herniosa, P. eiseni, P. lugubris, $P$. palustris and $P$. hyperborea. The latter species is most abundant, being presented in the material by slightly less than 1000 specimens.

The highest species number (73) among the studied habitats has been observed in the best studied tundra communities (Table 1). Yet, a high species diversity has been discovered in the spruce forests (66), the mountain birch forests (61) and the birch forests (43). All other habitats have a less number of species probably due to their poorer exploration. On the contrary, in the Swedish mountains (Torneträsk area) [Holm, 1950] and in the northernmost Finnish low mountains (the vicinity of Kevo Subarctic Research Station) [Koponen, 1976], the species number decreases with the increase of altitude (particularly when changing from the taiga to the tundra).

Nearly a half of the collected species (61) were recorded from the only habitat type. Some of the unique species for both the tundra and the spruce forest are most numerous amounting for 23\% (17 species) and $24 \%$ (16) respectively.

The majority of studied habitats were split into two main groups by means of the cluster analysis (Fig. 3). The first one includes the spider assemblages of the 
Table 1. Species composition and habitat distribution of the spiders in the Khibiny Mountains. Таблица 1. Видовой состав и биотопическое распределение пауков в Хибинских горах.

\begin{tabular}{|c|c|c|c|c|c|c|c|c|c|c|c|}
\hline Taxa & SF & BF & MB & $\mathbf{T}$ & SD & SR & B & $\mathbf{R}$ & $\mathbf{M}$ & PF & $\mathbf{A H}$ \\
\hline \multicolumn{12}{|l|}{ Fam. Amaurobiidae } \\
\hline Arctobius agelenoides (Emerton, 1919) > & & & & + & & & & & & & \\
\hline \multicolumn{12}{|l|}{ Fam. Araneidae } \\
\hline Cyclosa conica (Pallas, 1772) * > & & & & & & & & & & + & \\
\hline \multicolumn{12}{|l|}{ Fam. Gnaphosidae } \\
\hline Gnaphosa lapponum (L. Koch, 1866) * > & & & & + & & & & & & & \\
\hline G. muscorum (L. Koch, 1866) & & & & + & & & & & & & \\
\hline Haplodrassus signifer (C.L. Koch, 1839) & & & + & + & & & & & & & \\
\hline H. soerenseni (Strand, 1900) & + & & + & + & & & & & & & \\
\hline Micaria alpina L. Koch, 1872 & & & & + & & & & & & & \\
\hline Zelotes subterraneus (C.L. Koch, 1833) & & & + & & & & & & & & \\
\hline \multicolumn{12}{|l|}{ Fam. Hahniidae } \\
\hline Cryphroeca silvicola (C.L. Koch, 1834) & + & + & & & & & & & & + & \\
\hline Hahnia ononidum Simon, 1875 & + & + & & + & & & & & & & \\
\hline \multicolumn{12}{|l|}{ Fam. Linyphiidae } \\
\hline Abacoproeces saltuum (L. Koch, 1872) & + & & & & & & & & & & \\
\hline Abiskoa abiskoensis (Holm, 1945) > & + & & & & & & & & & & \\
\hline Agnyphantes expunctus (O. Pickard-Cambridge, 1875) & + & & & & & & & & & & \\
\hline Agyneta affinis (Kulczyński, 1898) * > & & & + & & & & & & & & \\
\hline A. cauta (O. Pickard-Cambridge, 1902) & + & & + & + & & & & & & & \\
\hline A. conigera $(\mathrm{O}$. Pickard-Cambridge, 1863) & + & & + & & & & & & & & \\
\hline A. gulosa (L. Koch, 1869) & & + & + & + & & + & & & & & \\
\hline A. rurestris $($ C.L. Koch, 1836) $*>$ & & & & + & & & & & & & \\
\hline Allomengea scopigera (Grube, 1859) & + & + & & + & & & & & & & \\
\hline Bathyphantes eumenis (L. Koch, 1879) & & + & & & & & & & & & \\
\hline B. reprobus (Kulczyński, 1916) > & + & & & & & & & & & & \\
\hline B. setiger F.O. Pickard-Cambridge, 1894 & & & & & & & & & & & + \\
\hline Bolephthyphantes index (Thorell, 1856) & & & + & + & & & & & & & \\
\hline Bolyphantes alticeps (Sundevall, 1833) & & & & & & & + & & & & \\
\hline B. luteolus (Blackwall, 1833) & + & + & & + & & & & & & & \\
\hline Centromerus arcanus (O. Pickard-Cambridge, 1873) & + & + & + & + & & & + & & & & \\
\hline C. incilium (L. Koch, 1881) > & & + & & & & & & & & & \\
\hline C. sylvaticus (Blackwall, 1841) & + & + & + & & & & & + & + & & + \\
\hline
\end{tabular}


Table 1 (contituing) Таблица 1 (продолжение)

\begin{tabular}{|c|c|c|c|c|c|c|c|c|c|c|c|}
\hline Ceraticelus bulbosus (Emerton, 1882) > & + & & & & & & & & & & \\
\hline Ceratinella brevipes (Westring, 1851) & + & & + & + & & & & & & & \\
\hline C. brevis (Wider, 1834) & + & & & & & & & & & & \\
\hline C. wideri (Thorell, 1871) *> & & & + & & & & & & & & \\
\hline Cnephalocotes obscurus (Blackwall, 1834) & & & + & + & & & & & & & \\
\hline Decipiphantes decipiens (L. Koch, 1879) & + & & + & + & & & & & & & \\
\hline Dicymbium nigrum (Blackwall, 1834) * > & & & & & & & & & + & & \\
\hline Diplocentria bidentata (Emerton, 1882) & & & + & + & & & & & & & \\
\hline D. rectangulata (Emerton, 1915) & + & & + & + & & & & & & & \\
\hline Diplostyla concolor (Wider, 1834) > & + & & & & & & & & & & \\
\hline Drapetisca socialis (Sundevall, 1833) * & & & & & & & & & & + & \\
\hline Drepanotylus borealis Holm, 1945 & & & & & & & & + & & & + \\
\hline Entelecara erythropus (Westring, 1851) *> & & & & + & & & & & & & \\
\hline Erigone arctica maritima Kulczyński, 1902 & & & & & & & & + & & & \\
\hline E. atra Blackwall, 1833 & & & & & + & & & + & + & & \\
\hline E. capra Simon, 1884 & & & & & & & + & + & & & \\
\hline E. dentipalpis (Wider, 1834) & & & & & & & & + & & & \\
\hline Estrandia grandaeva (Keyserling, 1886) & & + & & & & & & & & & \\
\hline Gnathonarium dentatum (Wider, 1834) > & & & & & & & & & & & + \\
\hline Gonatium rubens (Blackwall, 1833) & & & + & + & & & & & & & \\
\hline Hilaira herniosa (Thorell, 1875) & + & + & + & + & & & & + & & & \\
\hline H. nubigena Hull, 1911 & & & & & & & + & & & & \\
\hline Horcotes strandi (Sytshevskaja, 1935) > & & + & & & & & & & & & \\
\hline Hypomma bituberculatum (Wider, 1834) & & & & & & & & + & & & \\
\hline Hypselistes jacksoni (O. Pickard-Cambridge, 1902) & & & & + & & & + & & & & \\
\hline Improphantes complicatus (Emerton, 1882)> & + & & & & & & & & & & \\
\hline Incestophantes kochiellus (Strand, 1900) & + & & & & & & & & & & \\
\hline Kaestneria pullata (O. Pickard-Cambridge, 1863) & & + & & & & & & + & & & \\
\hline Leptorhoptrum robustum (Westring, 1851) & + & + & + & + & & + & & + & + & & + \\
\hline Macrargus carpenteri (O. Pickard-Cambridge, 1894) & & & & + & & & & & & & \\
\hline M. multesimus (O. Pickard-Cambridge, 1875) & & + & & & & & & & & & \\
\hline M. rufus (Wider, 1834) & + & + & + & + & & & & & & & \\
\hline Maro minutus O. Pickard-Cambridge, 1906 & + & & & + & & & & & & & \\
\hline M. sublestus Falconer, 1915 & & & & + & & & & & & & \\
\hline
\end{tabular}


Table 1 (contituing) Таблица 1 (продолжение)

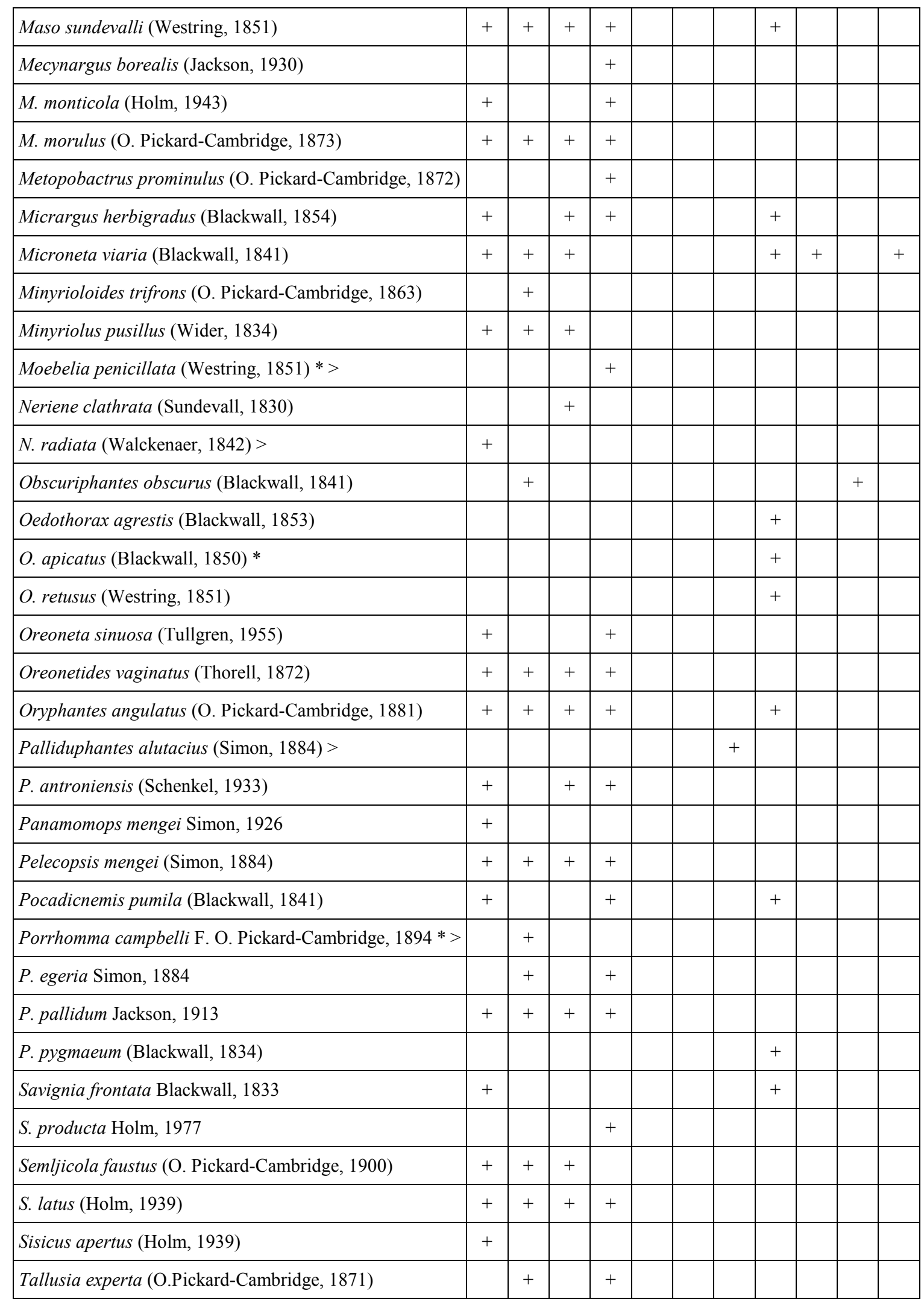


Table 1 (contituing) Таблица 1 (продолжение)

\begin{tabular}{|c|c|c|c|c|c|c|c|c|c|c|}
\hline Tapinocyba pallens (O. Pickard-Cambridge, 1872) & + & + & + & & & & & & & \\
\hline Tenuiphantes alacris (Blackwall, 1853) & + & + & + & + & & & & & & \\
\hline T. mengei (Kulczyński, 1887) & & & & & & & + & & & \\
\hline T. nigriventris (L. Koch, 1879) & + & + & + & & & & & + & & \\
\hline T. tenebricola (Wider, 1834) & + & + & + & + & & & + & + & & \\
\hline Tibioplus diversus (L. Koch, 1879) & + & & + & & & & & & & \\
\hline Tiso aestivus (L. Koch, 1872) & & & & + & & & & & & \\
\hline Walckenaeria capito (Westring, 1861) & & & + & + & & & & & & + \\
\hline W. cuspidata Blackwall, 1833 & + & & & + & & & & & & + \\
\hline W. dysderoides (Wider, 1834) & + & & + & & & & & & & \\
\hline W. karpinskii (O. Pickard-Cambridge, 1873) & + & + & + & + & & & & & & \\
\hline W. nudipalpis (Westring, 1851) & + & + & + & + & & + & & & & \\
\hline Zornella cultrigera (L. Koch, 1879) & + & + & + & + & & & & & & \\
\hline \multicolumn{11}{|l|}{ Fam. Liocranidae } \\
\hline Agroeca lusatica (L. Koch, 1875) *> & & & & + & & & & & & \\
\hline A. proxima (O. Pickard-Cambridge, 1871) & & & + & + & & & + & & & \\
\hline \multicolumn{11}{|l|}{ Fam. Lycosidae } \\
\hline Acantholycosa norvegica (Thorell, 1872) > & & & + & + & + & & & & & \\
\hline Alopecosa aculeata (Clerck, 1758) & & & + & + & & & & & & \\
\hline A. pinetorum (Thorell, 1856) $*>$ & & & + & + & & & & & & \\
\hline A. pulverulenta (Clerck, 1758) & & & & + & & & & & & \\
\hline A. taeniata (C.L. Koch, 1835) *> & + & & + & & & & & & & \\
\hline Arctosa alpigena (Doleschall, 1852) & & & & + & + & & & & & \\
\hline Pardosa amentata (Clerck, 1758) & + & & + & + & + & & + & + & & \\
\hline P. eiseni (Thorell, 1875) & & & + & + & & & & & & \\
\hline P. hyperborea (Thorell, 1872) & & & + & + & & & & & & \\
\hline P. lugubris (Walckenaer, 1802) & + & & + & + & & & & & & \\
\hline P. palustris (Linnaeus, 1758) & & & + & + & + & & & & & \\
\hline P. prativaga (L. Koch, 1870) & & & & + & & & & & & \\
\hline P. sphagnicola (Dahl, 1908) & & + & & + & & & & & & \\
\hline Trochosa terricola Thorell, 1856 & + & & + & & & & & & & \\
\hline Xerolycosa nemoralis (Westring, 1861) & & & + & & + & & & & & \\
\hline \multicolumn{11}{|l|}{ Fam. Philodromidae } \\
\hline Philodromus cespitum (Walckenaer, 1802) * > & & & & & & & & & + & \\
\hline Tibellus oblongus (Walckenaer, 1802) & + & & & & & & & & & \\
\hline
\end{tabular}


Table 1 (contituing) Таблица 1 (продолжение)

\begin{tabular}{|c|c|c|c|c|c|c|c|c|c|c|c|}
\hline Fam. Salticidae & & & & & & & & & & & \\
\hline Evarcha falcata (Clerck, 1758) & + & & & & & & & & & & \\
\hline Sitticus ranieri (Peckham et Peckham, 1909) & & & & + & & + & & & & & \\
\hline Talavera aequipes (O. Pickard-Cambridge, 1871) & & & & + & & & & & & & \\
\hline Fam. Tetragnathidae & & & & & & & & & & & \\
\hline Tetragnatha extensa (Linnaeus, 1758) & & + & & & & & & + & & & \\
\hline Fam. Theridiidae & & & & & & & & & & & \\
\hline Robertus lividus (Blackwall, 1836) & + & + & + & + & & & & + & + & & + \\
\hline R. lyrifer Holm, 1939 & + & & & & & & & & & & \\
\hline R. scoticus Jackson, 1914 & + & + & + & & & & & & & & \\
\hline Fam. Thomisidae & & & & & & & & & & & \\
\hline Ozyptila arctica Kulczyński, 1908 & & + & + & + & & & & & & & \\
\hline O. trux (Blackwall, 1846) & + & & + & & & & & & & & \\
\hline Xysticus audax (Schrank, 1803) & + & & & & & & & & & & \\
\hline X. luctuosus (Blackwall, 1836) & + & & + & & & & & & & & \\
\hline X. obscurus Collett, 1877 & & & + & + & & & & & & & \\
\hline X. sabulosus (Hahn, 1832) > & & & & & & & & & & & + \\
\hline Total species & 66 & 43 & 61 & 73 & 1 & 8 & 7 & 26 & 9 & 5 & 10 \\
\hline Total families & 8 & 6 & 6 & 9 & 1 & 3 & 1 & 5 & 3 & 4 & 3 \\
\hline Total unique species & 16 & 7 & 4 & 17 & $\mathbf{0}$ & 0 & 3 & 8 & 1 & 3 & 3 \\
\hline
\end{tabular}

The species that have been found in the Kola Peninsula from the Khibiny marked only are marked with $(>)$; the species that have been recorded from the Kola Peninsula for the first time are marked with (*). Abbreviations: SF — spruce forests; BF — birch forests; MB mountain birch forests; $\mathrm{T}$ - tundra communities; SD — stony desert; SR — screes; B — bogs; R — riparian habitats; M — meadows; $\mathrm{PF}$ - pine forests; $\mathrm{AH}$ - anthropogenic habitats.

Виды, найденные на Кольском полуострове только в Хибинах отмечены (>); виды, отмеченные впервые для Кольского полуострова выделены (*). Сокращения: SF — еловые леса; BF — березовые леса; МВ — березовые криволесья; Т — тундры; $\mathrm{SD}$ - каменистая пустыня; SR - каменистые осыпи; B — болота; R — прибрежные сообщества; M - луга; PF — coсновые леса; $\mathrm{AH}$ - антропогенные местообитания.

main types of mountain communities (mountain birch forests, spruce forests, tundra communities, birch forests), whereas the other one - the habitats that not depend on altitude (meadows, riparian and anthropogenic habitats). The less studied sites (screes, pine forests, stony desert, bogs) turned out to be separated on individual branches on the dendrogram. Nine species (Macrargus rufus, Mecynargus morulus, Oreonetides vaginatus, Pelecopsis mengei, Porrhomma pallidum, Semljicola latus, Tenuiphantes alacris, Walckenaeria karpinskii, Zornella cultrigera) were recorded in each type of the mountain habitats and were not found in other biotopes. Both Leptorhoptrum robustum and Robertus lividus were found in almost all the studied habitats (except for stony desert, bogs, pine forest and also screes for the latter species).
Despite the fact that the number of arachnological studies of the Khibiny Mts has increased recently, the data on spider distribution in this region are still incomplete. The number of spider species found there is lesser than that of the adjacent, well-studied Torneträsk Mts (Sweden), from where there are as many as 190 recorded species [Holm, 1950; T. Kronestedt, pers. comm.]. Data on the spider fauna of some areas of the Khibiny still remain poor or are absent.

ACKNOWLEDGEMENTS. I'm very grateful to Irina Zenkova, Viktoriya Pozharskaya, and Ivan Nekhaev whose materials have been used in this paper. Special thanks go to Ilya Kamayev who provided me with additional data. I am deeply obliged to Ivan Nekhaev for useful comments and the comprehensive help in the preparation of this paper, to An- 


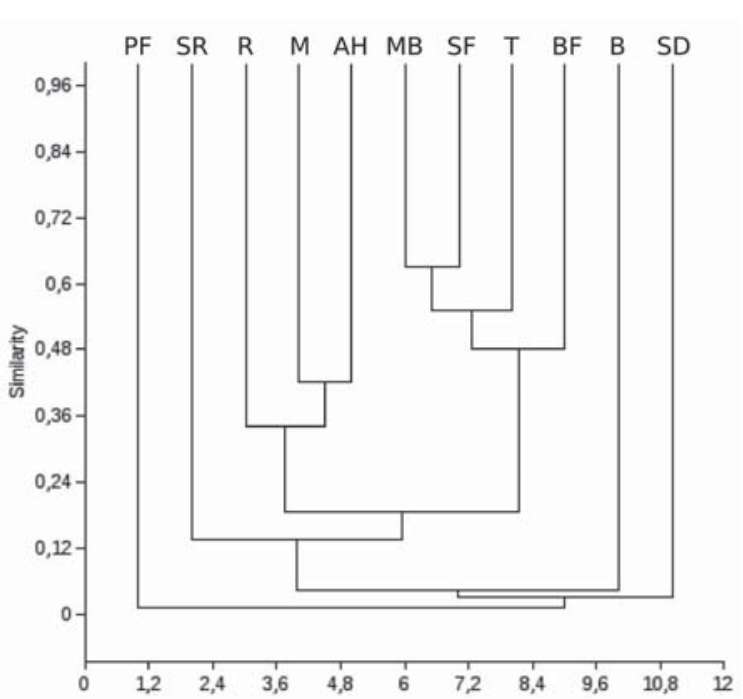

Fig. 3. A similarity dendrogram of the different habitats by their species composition: SF — spruce forests; BF — birch forests; MB - mountain birch forests; $\mathrm{T}$ - tundra communities; SD — stony desert; SR — screes; B — bogs; R - riparian habitats; $\mathrm{M}$ - meadows; $\mathrm{PF}$ - pine forests; $\mathrm{AH}$ - anthropogenic habitats.

Рис. 3. Дендрограмма сходства местообитаний разных типов по видовому составу: SF - еловые леса; BF - березовые леса; MB - березовые криволесья; Т - тундры; SD - каменистая пустыня; SR - каменистые осыпи; В - болота; R прибрежные сообщества; M - луга; PF — сосновые леса; AH антропогенные местообитания.

drei Tanasevitch for his consultation on the spider taxonomy and distribution, to Dmitri Logunov and Yuri Marusik for critical remarks on the earlier draft. I am also thankful to Kirill Mikhailov for the helpful information about spider collections, to Torbjörn Kronestedt who helped in obtaining some papers, and to Tatyana Chernenkova and Eugene Borovichev for their help with plant identification. The study was supported in part by the Russian Foundation for Basic Research (RFFI), grants \# 15-04-05964 A and \# 12-04-01538-a.

\section{References}

Azarkina G.N., Logunov D.V. 2001. Separation and distribution of Xysticus cristatus (Clerck, 1758) and X. audax (Schrank, 1803) in eastern Eurasia, with description of a new species from the mountains of Central Asia (Aranei: Thomisidae) // Arthropoda Selecta. Vol.9 (for 2000). No.2. P.133-150.

Blagoev G.A., Nikolova N.I., Sobel C.N., Hebert P.D., Adamowicz S.J. 2013. Spiders (Araneae) of Churchill, Manitoba: DNA barcodes and morphology reveal high species diversity and new Canadian records // BMC Ecology. Vol.13. No.44. P.1-17.

Buckle D.J., Carroll D., Crawford R.L., Roth V.D. 2001. Linyphiidae and Pimoidae of America north of Mexico: checklist, synonymy, and literature // Fabréries. Suppl.10. P.89-191.

Dondale C.D., Redner J.H. 1975. The genus Ozyptila in North America (Araneida, Thomisidae) // J. Arachnol. Vol.2. No.3. P.129-181.

Dondale C.D., Redner J.H. 1978. The insects and arachnids of Canada, Part 5. The crab spiders of Canada and Alaska, Araneae: Philodromidae and Thomisidae. Ottawa: Research Branch, Agriculture Canada. 255 p.

Dondale C.D., Redner J.H. 1990. The insects and arachnids of Canada, Part 17. The wolf spiders, nurseryweb spiders, and lynx spiders of Canada and Alaska, Araneae: Lycosidae, Pisauridae, and Oxyopidae. Ottawa: Research Branch, Agriculture Canada. 383 p.

Eskov K.Y. 1994. Catalogue of the linyphiid spiders of northern Asia (Arachnida, Araneae, Linyphiidae). Sofia-Moscow: Pensoft Publ. 144 p.

Esyunin S.L. 2006. Remarks on the Ural spider fauna (Arachnida: Aranei). 11. New faunistic records // Arthropoda Selecta. Vol.14 (for 2005). No.4. P.329-337.

Esyunin S.L., Efimik V.E. 1996. Catalogue of the spiders (Arachnida, Aranei) of the Urals. Moscow: KMK Scientific Press Ltd. 229 p.

Esyunin S.L., Ermakov A.I., Mikhailov Yu.E. 2013. Remarks on the Ural spider fauna (Arachnida: Aranei), 14. On the spider fauna of the Kytlym plexus of mountains (the North Urals) // Arthropoda Selecta. Vol.22. No.1. P.75-82.

Fedotov D.M. 1912. [To the spider fauna of Murman and Novaya Zemlya] // Annu. Mus. Zool. Acad. Sci. St.-Petersb. T.16. No.4. P.449-474. [in Russian].

Fridolin V.Yu. 1936. [Animal-plant community of the Khibiny Mountain country]. Moscow-Leningrad: AN USSR Publ. 295 p. [in Russian].

Hammer Ø., Harper D.A.T., Ryan P.D. 2001. PAST: Paleontological statistics software package for education and data analysis / / Palaeontologia Electronica. V.4. No.1. P.1-9.

Holm Å. 1950. Studien über die Spinnenfauna des Torneträskgebietes // Zoologiska Bidrag från Uppsala. Bd. 29. P.103-213.

Ivie W. 1969. North American spiders of the genus Bathyphantes (Araneae, Linyphiidae) // Amer. Mus. Novit. No.2364. P.170 .

Kamayev I.O. 2010. [Population of spiders (Aranei) at the highaltitude gradient of the Khibiny] // Lomonosov-2010: Mezhdunarodnaya konferenciya studentov, aspirantov i molodykh uchenykh; sekciya "Biologiya". Moscow: MAKS Press. P.126127. [in Russian].

Koponen S. 1976. Spider fauna (Araneae) of Kevo area, northernmost Finland // Rep. Kevo Subarctic Research Station. Vol.13. P.48-62.

Koponen S. 1993. On the biogeography and faunistics of European spiders: latitude, altitude and insularity // Bulletin de la Société Neuchâteloise des Sciences Naturelles. Vol.116. No.1. p.141-152.

Koroleva N.E. 2001. [Syntaxonomical survey of mountain tundra vegetation of Khibiny] // Byulleten Moscovskogo Obshchestva Ispytatelei Prirody. Otdel Biologicheskii. Vol.106. No.4. P.5057. [in Russian, with English summary].

Kremenetski K.V., Boettger T., MacDonald G.M., Vaschalova T., Sulerzhitsky L., Hiller A. 2004. Medieval climate warming and aridity as indicated by multiproxy evidence from the Kola Peninsula, Russia // Palaeogeography, Palaeoclimatology, Palaeoecology. Vol.209. Issues 1-4. P.113-125.

Kronestedt T., Logunov D.V. 2001. On the separation of Sitticus ranieri Peckham \& Peckham and S. saxicola (C.L. Koch) (Araneae, Salticidae) // Rev. suisse Zool. T.108. Fasc.3. P.463-481.

Kronestedt T., Marusik Yu.M., Omelko M.M. 2014. Studies on species of Holarctic Pardosa groups (Araneae, Lycosidae). VIII. The Palaearctic species of the Pardosa nigra group // Zootaxa. Vol.3894. No.1. P.33-60.

Leech R. E. 1972. A revision of the Nearctic Amaurobiidae (Arachnida: Araneida) // Mem. Entomol. Soc. Canada. No.84. P.1182.

Logunov D.V., Marusik Yu.M. 2000. Catalogue of the jumping spiders of northern Asia (Arachnida, Araneae, Salticidae). Moscow: KMK Scientific Press Ltd. 299 p.

Locket G.H. 1964. Type material of British spiders in the O. Pickard-Cambridge collection at Oxford // Ann. Mag. Nat. Hist. Ser.13. Vol.7. P.257-278.

Marusik Yu.M., Alfimov A.V. 2012. [Spiders (Arachnida, Aranei) and microclimate of xeromorphic habitats in the lower reaches of Kolyma River $\left.69^{\circ} \mathrm{N}\right] / /$ Euroasian Entomological J. T.11. Suppl.1. P.25-38. [in Russian, with English summary].

Marusik Yu.V., Böcher J., Koponen S. 2006. The collection of Greenland spiders (Aranei) kept in the Zoological Museum, 
University of Copenhagen // Arthropoda Selecta. Vol.15. No.1. P.59-80.

Marusik Y.M., Buckle D.J., Koponen S. 2007. A survey of the Holarctic Linyphiidae (Araneae), a review of the erigonine genus Zornella Jackson, 1932 // Acta Zootaxonomica Sinica. Vol. 32. No.1. P.21-34.

Marusik Yu.M., Eskov K.Yu. 2009. [Spiders (Arachnida: Aranei) of the tundra zone of Russia] // Vidy i soobshchestva v ekstremalnykh usloviyakh. Moscow - Sofia: KMK Scientific Press PENSOFT Publ. P.92-123 [in Russian, with English summary].

Marusik Yu.M., Koponen S. 2005. A survey of spiders (Araneae) with Holarctic distribution // J. Arachnol. Vol.33. No.2. P.300305.

Marusik Yu.M., Logunov D.V., Koponen S. 2000. Spiders of Tuva, South Siberia. Magadan: IBPN FEB RAS. 252 p.

Marusik Y.M., Omelko M.M. 2014. A survey of East Palaearctic Gnaphosidae (Araneae). 3. On new and poorly known Gnaphosa Latreille, 1804 // Zootaxa. Vol.3894. No.1. P.10-32.

Mikhailov K.G. 2013. The spiders (Arachnida: Aranei) of Russia and adjacent countries: a non-annotated checklist // Arthropoda Selecta. Suppl. No.3. P.1-262.

Mishkin B.A. 1953. [The flora of the Khibiny Mountains, its analysis and history]. Moscow-Leningrad: AN SSSR Publ. 113 p. [in Russian].

Ovtsharenko V.I., Platnick N.I., Song D.X. 1992. A review of the North Asian ground spiders of the genus Gnaphosa (Araneae, Gnaphosidae) // Bull. Amer. Mus. Nat. Hist. No.212. P.1-88.

Palmgren P. 1975. Die Spinnenfauna Finnlands und Ostfennoskandiens VI. Linyphiidae $1 / /$ Fauna Fennica. T.28. P.1-102.

Palmgren P. 1976. Die Spinnenfauna Finnlands und Ostfennoskandiens. VII. Linyphiidae 2 // Fauna Fennica. T.29. P.1-126.

Palmgren P. 1977. Die Spinnenfauna Finnlands und Ostfennoskandiens. VIII. Argyronetidae, Agelenidae, Hahniidae, Dictynidae, Amaurobiidae, Titanoecidae, Segestriidae, Pholcidae, Sicariidae // Fauna Fennica. T.30. P.1-50.

Platnick N.I., Dondale C.D. 1992. The insects and arachnids of Canada, Part 19. The ground spiders of Canada and Alaska (Araneae: Gnaphosidae). Ottawa: Research Branch, Agriculture Canada Publication. 297 p.

Rybalov L.B., Kamayev I.O. 2012. Comparative analysis and longterm dynamics of soil macrofauna in forest-tundra ecotone of the Khibiny mountains // Russian Entomol. J. Vol.21. No.2. P.179-183.

Saaristo M. 1971. Revision of the genus Maro O.P.-Cambridge (Araneae, Linyphiidae) // Ann. Zool. Fennici. Vol.8. P.463-482.

Saariato M.I., Eskov K.Y. 1996. Taxonomy and zoogeography of the hypoarctic erigonine spider genus Semljicola (Araneida, Linyphiidae) // Acta Zool. Fennica. No.201. P.47-69.

Shlyakov R.N. 1961. [The flora of leafy mosses in the Khibiny Mountains]. Murmanskoe knizhnoe izdatelstvo. 249 p. [in Russian].
Tanasevitch A.V. 2008. New records of linyphiid spiders from Russia, with taxonomic and nomenclatural notes (Aranei: Linyphiidae) // Arthropoda Selecta. Vol.16. No.2. P.115-135.

Tanasevitch A.V. 2010. On the synonymy of linyphiid spiders of the Russian fauna (Arachnida: Aranei: Linyphiidae). 1 // Arthropoda Selecta. Vol.19. No.4. P.273-282.

Tanasevitch A. V. 2011. On the synonymy of linyphiid spiders of the Russian fauna (Arachnida: Aranei: Linyphiidae). 2 // Arthropoda Selecta. Vol.20. No.2. P.129-143.

Tanasevitch A.V. 2013. The linyphiid spiders of the Altais, southern Siberia (Aranei: Linyphiidae) // Arthropoda Selecta. Vol.22. No.3. P.267-306

Tanasevitch A.V. 2014. Linyphiid spiders of the world. Online at http://www.andtan.newmail.ru/list/index.htm

Tanasevitch A.V., Kamayev I.O. 2011. [Spiders of the Kola Peninsula, Russia (Arachnida: Aranei)] // Caucasian Entomol. Bull. Vol.7. No.1. P.7-32 [in Russian, with English summary].

Tanasevitch A.V., Koponen S. 2007. Spiders (Aranei) of the southern tundra in the Russian Plane // Arthropoda Selecta. Vol.15 (for 2006). No.4. P.295-345.

Tanasevitch A.V., Rybalov L.B. 2010. On spiders from the tundra zone of the Kola Peninsula, Russia (Arachnida: Aranei) // Arthropoda Selecta. Vol.19. No.1. P.41-56.

Tuneva T.K. 2006. Review of the family Gnaphosidae in the Ural fauna (Aranei), 5. Genera Micaria Westring, 1851 and Arboricaria Bosmans, 2000 // Arthropoda Selecta. Vol.15. No.3. P.229-250.

Vasilyeva A.A., Nekhaev I.O. 2009. [To the ecology of spider Pardosa palustris (Aranei, Lycosidae) in Murmansk Area] // Problemy morskoy paleoekologii i biogeografii v epokhu globalnikh izmeneniy. Moscow: GEOS. P.33-34. [in Russian].

World Spider Catalog 2015. World Spider Catalog. Natural History Museum Bern, online at http://wsc.nmbe.ch, version 16, accessed on 11.01.2015.

Yakovlev B.A. 1961. [The climate of Murmansk Region]. Murmansk: Murmanskoe knizhnoe izdatelstvo. 199 p. [in Russian].

Zenkova I.V., Pozharskaya V.V., Pokhilko A.A. 2009. [Materials to the soil fauna of the Khibiny Mountain massive on a example of Vudyavrchorr Mt.] // Proceedings of the Murmansk State Technical University. Vol.12. No.3. P.516-524. [in Russian, with English summary].

Zenkova I.V., Zaitsev A.S., Zalish L.V., Liskovaya A.A. 2011. [A list of the oribatid mites (Acariformes: Oribatida) in the tundra and northern taiga soils of the Murmansk region] // Trudy Karelskogo nauchnogo centra RAN. No.1. P.54-67. [in Russian, with English summary].

Zenkova I.V., Kolesnikova A.A., Filippov B.Yu., Vershinina S.D., Trushitsina O.S., Nekhaeva A.A. 2013. Materials on the fauna of beetles and spiders of the 'Pasvik' Reserve mountain system // Zelenyi poyas Fennoskandii: materialy mezhdunarodnoi nauchno-prakticheskoi konferencii (Petrozavodsk, 7-12 oktyabrya 2013). Petrozavodsk: KarNC RAN. P.138-139.

Responsible editor D.V. Logunov 